\title{
The Myopic Stable Set for Social Environments
}

Citation for published version (APA):

Demuynck, T., Herings, P. J-J., Saulle, R., \& Seel, C. (2019). The Myopic Stable Set for Social

Environments. Econometrica, 87(1), 111-138. https://doi.org/10.3982/ECTA14954

Document status and date:

Published: 01/01/2019

DOI:

10.3982/ECTA14954

Document Version:

Publisher's PDF, also known as Version of record

Document license:

Taverne

\section{Please check the document version of this publication:}

- A submitted manuscript is the version of the article upon submission and before peer-review. There can be important differences between the submitted version and the official published version of record.

People interested in the research are advised to contact the author for the final version of the publication, or visit the DOI to the publisher's website.

- The final author version and the galley proof are versions of the publication after peer review.

- The final published version features the final layout of the paper including the volume, issue and page numbers.

Link to publication

\footnotetext{
General rights rights.

- You may freely distribute the URL identifying the publication in the public portal. please follow below link for the End User Agreement:

www.umlib.nl/taverne-license

Take down policy

If you believe that this document breaches copyright please contact us at:

repository@maastrichtuniversity.nl

providing details and we will investigate your claim.
}

Copyright and moral rights for the publications made accessible in the public portal are retained by the authors and/or other copyright owners and it is a condition of accessing publications that users recognise and abide by the legal requirements associated with these

- Users may download and print one copy of any publication from the public portal for the purpose of private study or research.

- You may not further distribute the material or use it for any profit-making activity or commercial gain

If the publication is distributed under the terms of Article $25 \mathrm{fa}$ of the Dutch Copyright Act, indicated by the "Taverne" license above, 


\title{
THE MYOPIC STABLE SET FOR SOCIAL ENVIRONMENTS
}

\author{
THOMAS DEMUYNCK \\ Ecares, Université Libre de Bruxelles \\ P. JEAN-JACQUES HERINGS \\ Department of Economics, Maastricht University \\ RICCARDO D. SAULLE \\ Department of Economics, Maastricht University \\ CHRISTIAN SEEL \\ Department of Economics, Maastricht University
}

\begin{abstract}
We introduce a new solution concept for models of coalition formation, called the myopic stable set (MSS). The MSS is defined for a general class of social environments and allows for an infinite state space. An MSS exists and, under minor continuity assumptions, it is also unique.

The MSS generalizes and unifies various results from more specific applications. It coincides with the coalition structure core in coalition function form games when this set is nonempty; with the set of stable matchings in the Gale-Shapley matching model; with the set of pairwise stable networks and closed cycles in models of network formation; and with the set of pure strategy Nash equilibria in pseudo-potential games and finite supermodular games. We also characterize the MSS for the class of proper simple games.
\end{abstract}

KEYWORDS: Social environments, group formation, stability, Nash equilibrium.

\section{INTRODUCTION}

MODELS OF COALITION FORMATION study a widespread and important pattern of human interaction: agents tend to form groups of equally interested individuals, but behave in a noncooperative way toward outsiders. For example, individuals in a community join forces to provide a local public good, voters create parties to attain their political goals, and firms set up lobby groups to influence policy-makers.

The literature studies coalition formation in many distinct settings, like networks, coalition function form games, and matching models. In this paper, we focus on a general class of social environments that covers all of these settings and many more. More precisely, we define a social environment on the basis of four components (Chwe (1994)): a finite collection of agents, a set of states, for every agent preferences over the set of states, and an effectivity correspondence that models the feasible transitions from one state to another. We only require that the set of states is a nonempty, compact metric space. As such, in contrast to most settings in the literature, we allow the state space to be infinite.

Thomas Demuynck: Thomas.Demuynck@ulb.ac.be

P. Jean-Jacques Herings: P.Herings@maastrichtuniversity.nl

Riccardo D. Saulle: R.Saulle@maastrichtuniversity.nl

Christian Seel: C.Seel@maastrichtuniversity.nl

We thank four anonymous referees for useful comments on earlier versions of this paper. Thomas Demuynck acknowledges financial support by the Fonds de la Recherche Scientifique-FNRS under grant F.4516.18. 
For such social environments, we define a new solution concept called the myopic stable set, abbreviated as MSS. The MSS extends the idea of level- 1 farsighted stability by Herings, Mauleon, and Vannetelbosch $(2009,2018)$ from finite networks to the general class of social environments. The MSS is defined by three conditions, deterrence of external deviations, asymptotic external stability, and minimality. Deterrence of external deviations requires that no coalition benefits by deviating from a state inside the MSS to a state outside the MSS. Asymptotic external stability makes sure that from any state outside the MSS it is possible to get arbitrarily close to a state inside by a sequence of coalitional deviations. Finally, minimality requires that the MSS is minimal with respect to set inclusion.

Our notion of dominance is myopic as agents or coalitions do not predict how their decision to change the current state will lead to further changes by other agents or coalitions. Such a notion is natural in complex social environments where the number of possible states is large and agents have little information about the possible actions other agents may take or the incentives of other agents.

The myopic stable set is closely related to other myopic concepts as the core and the Nash equilibrium. It differs from the ones in the literature that focus on farsightedness (see among others, Chwe (1994), Herings, Mauleon, and Vannetelbosch $(2009,2018)$, Page, Wooders, and Kamat (2005), Page and Wooders (2009), Ray and Vohra (2015)), notions which strengthen farsightedness by using state-dependent expectations (Greenberg (1990), Xue (1998), Dutta and Vohra (2017)), and approaches to farsightedness that are inspired by noncooperative game theory (Herings, Mauleon, and Vannetelbosch (2004), Dutta, Ghosal, and Ray (2005)). In particular, Greenberg's (1990) optimistic and conservative standards of behavior are popular farsighted notions that, as our MSS, cover a large set of applications.

Our first main result (Theorem 3.1) shows that every social environment contains at least one nonempty MSS. Moreover, under minor continuity assumptions, we establish uniqueness of the MSS (Theorem 3.4). The existence and uniqueness results differ from many other popular solution concepts in the literature. For instance, the core and the coalition structure core for coalition function form games can be empty (Bondareva (1963), Scarf (1967), Shapley (1967)), the von Neumann-Morgenstern stable set may fail to exist or to be unique (Lucas $(1968,1992)$ ), optimistic and conservative standards of behavior might fail to exist (Greenberg (1990)), and the set of pure strategy Nash equilibria may be empty.

We provide several additional results that provide more insights about the structure of an MSS. For finite state spaces, we fully characterize the MSS as the union of all closed cycles (Theorem 3.9), that is, subsets which are closed under coalitional better replies. This result also provides a connection to stochastic processes of coalition and network formation as in Jackson and Watts (2002) and Sawa (2014). For infinite spaces, the union of all closed cycles is found to be a subset of the MSS. This result is helpful in applications and in the comparison to other solution concepts. For instance, any state in the core is a closed cycle and is therefore included in the MSS. As a special case of this result, it follows that the MSS contains the set of pure strategy Nash equilibria in a normal-form game. Next, we define a generalization of the weak improvement property (Friedman and Mezzetti (2001)) to social environments and we show that, under weak continuity conditions, the weak improvement property characterizes the collection of social environments for which the MSS coincides with the core. We also show that if the von Neumann-Morgenstern stable set exists, it has a nonempty intersection with any MSS.

We demonstrate the versatility of our results by analyzing the relationship between the MSS and other solution concepts in specific social environments. In particular, we show 
that the MSS coincides with the coalition structure core for coalition function form games (Kóczy and Lauwers (2004)) whenever the coalition structure core is nonempty; with the set of stable matchings in the one-to-one matching model by Gale and Shapley (1962); with the set of pairwise stable networks and closed cycles in models of network formation (Jackson and Watts (2002)); and with the set of pure strategy Nash equilibria in pseudopotential games (Dubey, Haimanko, and Zapechelnyuk (2006)) and finite supermodular games (Topkis (1979) and Milgrom and Roberts (1990)). We fully characterize the MSS for the class of proper simple games.

The structure of the paper is as follows. Section 2 provides the primitives of our general framework of social environments and introduces and motivates the MSS. Section 3 establishes existence, nonemptiness, and uniqueness results. Section 4 analyzes our solution concept for various settings and relates it to other stability concepts from the literature. Section 5 is a conclusion. All proofs can be found in the Appendix. Additional material is covered in the Online Supplemental Material (Demuynck, Herings, Saulle, and Seel (2019)).

\section{THE MYOPIC STABLE SET}

In this section, we first introduce the concept of a social environment. Next, we introduce the notions of dominance and asymptotic dominance, which we then use to define our solution concept, the myopic stable set.

Let $N$ be a nonempty finite set of individuals. A coalition $S$ is a subset of $N$. The set of nonempty subsets of $N$ is denoted by $\mathcal{N}$. Let $(X, d)$ be a metric space, where $X$ denotes our nonempty state space and $d$ is a metric on $X .{ }^{1}$ Let some state $x \in X$ be given and let $\varepsilon \in \mathbb{R}_{++}$. We define $B_{\varepsilon}(x)=\{y \in X \mid d(x, y)<\varepsilon\}$ as the open ball around $x$ with radius $\varepsilon$. The set $B_{\varepsilon}(x)$ contains all states in $X$ that are in an $\varepsilon$-neighborhood of $x$. For a sequence $\left(x^{k}\right)_{k \in \mathbb{N}}$ in $X$, we write $x^{k} \rightarrow x$ if for all $\varepsilon>0$, there is a number $N \in \mathbb{N}$ such that for all $k \geq N, x^{k} \in B_{\varepsilon}(x)$, that is, the sequence $\left(x^{k}\right)_{k \in \mathbb{N}}$ converges to $x$.

An effectivity correspondence $E$ associates with each pair of states $(x, y) \in X \times X$ a, possibly empty, collection of coalitions $E(x, y) \subseteq \mathcal{N}$. If $S \in E(x, y)$, we say that coalition $S$ can move from state $x$ to state $y$. If $E(x, y)=\emptyset$, then no coalition can move from $x$ to $y$.

Each individual $i \in N$ has a complete and transitive preference relation $\succeq_{i}$ over the state space $X$. The profile $\left(\succeq_{i}\right)_{i \in N}$ then lists the preferences of all individuals in $N$. We denote the asymmetric part of $\succeq_{i}$ by $\succ_{i}$, that is, $x \succ_{i} y$ if and only if $x \succeq_{i} y$ and not $y \succeq_{i} x$. A social environment is now defined as follows.

DEFINITION 2.1—Social Environment: A social environment is a tuple

$$
\Gamma=\left(N,(X, d), E,\left(\succeq_{i}\right)_{i \in N}\right)
$$

consisting of a nonempty, finite set of agents $N$, a nonempty, compact metric space $(X, d)$ of states, an effectivity correspondence $E$ on $X$, and a profile of preference relations $\left(\succeq_{i}\right)_{i \in N}$ over $X$.

In Section 4, we specify different social environments which correspond to applications such as coalition function form games, one-to-one matching, network formation, and noncooperative normal-form games.

\footnotetext{
${ }^{1}$ A metric is a function $d: X \times X \rightarrow \mathbb{R}_{+}$such that (i) for every $x, y \in X: d(x, y)=0$ if and only if $x=y$, (ii) for every $x, y \in X: d(x, y)=d(y, x)$, and (iii) for every $x, y, z \in X, d(x, y) \leq d(x, z)+d(z, y)$.
} 
For a given social environment $\Gamma=\left(N,(X, d), E,\left(\succeq_{i}\right)_{i \in N}\right)$, we say that a state $y \in X$ dominates another state $x \in X$ if there is a coalition such that (i) it can move from $x$ to $y$ and (ii) each of its members strictly prefers $y$ over $x$.

DEFINITION 2.2-Dominance: A state $y \in X$ dominates a state $x \in X$ under $E$ if there exists a coalition $S \in E(x, y)$ such that for every $i \in S$ it holds that $y \succ_{i} x$.

An alternative notion is the one of weak dominance. A state $y \in X$ weakly dominates a state $x \in X$ if there exists a coalition $S \in E(x, y)$ such that for all $i \in S, y \succeq_{i} x$ and there is at least one $j \in S$ such that $y \succ_{j} x$. When we restrict ourselves to settings with a finite state space $X$, all theoretical results from this section remain valid when we replace dominance by weak dominance. For settings where $X$ is infinite, most results remain valid with the exception of Theorem 3.7 below, which provides sufficient conditions for uniqueness.

Let some state $x \in X$ be given. The subset of $X$ consisting of all states that dominate $x$ together with the state $x$ itself is denoted by $f(x)$, so

$$
f(x)=\{x\} \cup\{y \in X \mid y \text { dominates } x \text { under } E\} .
$$

We refer to $f$ as the dominance correspondence. We define the two-fold composition of $f$ by $f^{2}(x)=\{z \in X \mid \exists y \in X: y \in f(x)$ and $z \in f(y)\}$. By induction, we can define the $k$ fold iteration $f^{k}(x)$ as the subset of $X$ that contains all states obtained by a composition of dominance correspondences of length $k \in \mathbb{N}$, that is, $y \in f^{k}(x)$ if there is a $z \in X$ such that $y \in f(z)$ and $z \in f^{k-1}(x)$. Since by definition $x \in f(x)$, it holds that, for all $k, \ell \in \mathbb{N}$, if $k \leq \ell$, then $f^{k}(x) \subseteq f^{\ell}(x)$. We define the set of all states that can be reached from $x$ by a finite number of dominations by $f^{\mathbb{N}}(x)$, so

$$
f^{\mathbb{N}}(x)=\bigcup_{k \in \mathbb{N}} f^{k}(x) .
$$

A state $y$ is said to asymptotically dominate the state $x$ if starting from $x$ it is possible to get arbitrarily close to $y$ in a finite number of dominations.

DEFINITION 2.3-Asymptotic Dominance: A state $y \in X$ asymptotically dominates a state $x \in X$ under $E$ if for all $\varepsilon>0$ there exists $k \in \mathbb{N}$ and a state $z \in f^{k}(x)$ such that $z \in B_{\varepsilon}(y)$.

We denote by $f^{\infty}(x)$ the set of all states in $X$ that asymptotically dominate $x$. Formally, we have

$$
f^{\infty}(x)=\left\{y \in X \mid \forall \varepsilon>0, \exists k \in \mathbb{N}, \exists z \in f^{k}(x) \text { such that } z \in B_{\varepsilon}(y)\right\} .
$$

It is easy to see that the set $f^{\infty}(x)$ coincides with the closure of the set $f^{\mathbb{N}}(x)$.

We are now ready to define our solution concept, the myopic stable set, abbreviated as MSS.

Definition 2.4-Myopic Stable Set: Let $\Gamma=\left(N,(X, d), E,\left(\succeq_{i}\right)_{i \in N}\right)$ be a social environment. The set $M \subseteq X$ is a myopic stable set if it is closed and satisfies the following three conditions:

1. Deterrence of external deviations: For all $x \in M, f(x) \subseteq M$.

2. Asymptotic external stability: For all $x \notin M, f^{\infty}(x) \cap M \neq \emptyset$.

3. Minimality: There is no closed set $M^{\prime} \subsetneq M$ that satisfies Conditions 1 and 2 . 
The solution concept that results when we replace dominance by weak dominance is called weak dominance MSS.

Let $M$ be an MSS. Deterrence of external deviations requires that no coalition of myopic agents can profitably deviate to a state outside $M$. Next, asymptotic external stability requires that from any state outside $M$ it is possible to get arbitrarily close to a state in $M$ by a finite number of myopic deviations. Observe that the empty set would necessarily violate asymptotic external stability, so any MSS is nonempty.

Although the property of asymptotic external stability resembles a notion of farsightedness, there is an important distinction. In models with farsighted behavior, coalitions deviate from the current state because they expect to profit from a move in some future period, that is, after possible subsequent moves by other coalitions. Our definition of asymptotic external stability, however, is myopic in the sense that coalitions deviate only because they see an immediate gain, without anticipating potential future deviations. The condition of deterrence of external deviations also reflects myopic behavior. Myopic agents will not leave the set as this would not result in an immediate gain for all players in a deviating coalition. In contrast, farsighted agents might do so in the hope of gains after subsequent deviations.

Finally, minimality imposes that there is no smaller closed set of states that satisfies deterrence of external deviations and asymptotic external stability. We do not impose a condition of internal stability in the sense of von Neumann and Morgenstern, so it is possible that a state in the MSS is dominated by another state in the MSS. The MSS is therefore stable as a set, whereas a state within the MSS may not be stable by itself.

For finite state spaces, the restriction imposed by asymptotic external stability remains unchanged if $f^{\infty}$ is replaced by $f^{\mathbb{N}}$. We refer to the property that for all states $x \notin M$, $f^{\mathbb{N}} \cap M \neq \emptyset$ as iterated external stability. For infinite state spaces, the two concepts differ. If one uses iterated external stability instead of asymptotic external stability, we show in the online supplement that a MSS might fail to exist.

A MSS is a minimal set satisfying deterrence of external deviations and asymptotic external stability. Dropping the minimality requirement leads to the concept of a quasi myopic stable set (QMSS) which is useful in the proofs.

Definition 2.5-Quasi Myopic Stable Set: Let $\Gamma=\left(N,(X, d), E,\left(\succeq_{i}\right)_{i \in N}\right)$ be a social environment. The set $M \subseteq X$ is a quasi myopic stable set if it is closed and satisfies deterrence of external deviations and asymptotic external stability.

\section{GENERAL PROPERTIES}

This section establishes existence of the myopic stable set in general and, under weak additional assumptions, its uniqueness. We also derive some additional structural properties of myopic stable sets that are used in the next section. We provide a brief discussion of the relationship between the MSS and the von Neumann-Morgenstern stable set. Finally, we relate our approach to dynamic models that rely on stochastic processes.

\subsection{Existence and Uniqueness}

The first main result is about existence of the myopic stable set.

THEOREM 3.1-Existence: Let $\Gamma$ be a social environment. Then a MSS exists. 
Note that the set of all states is a QMSS and the collection of all sets of states that are a QMSS is partially ordered by inclusion. In the proof of Theorem 3.1, we verify that the partially ordered collection satisfies the conditions for Zorn's lemma and apply it to conclude that there is a minimal QMSS, that is, a MSS.

Having established existence of a MSS, we now turn to the cardinality of such sets. The following lemma shows that two myopic stable sets cannot be disjoint.

LEMMA 3.2: Let $\Gamma$ be a social environment and let $M_{1}$ and $M_{2}$ be two myopic stable sets of $\Gamma$. Then $M_{1} \cap M_{2} \neq \emptyset$.

Suppose the intersection $M_{1} \cap M_{2}$ satisfies deterrence of external deviations and asymptotic external stability, that is, it is a QMSS. Then, by minimality of $M_{1}$ and $M_{2}$, the MSS is unique. In the online supplement, we show that for infinite social environments, the intersection $M_{1} \cap M_{2}$ might not satisfy asymptotic external stability without any additional assumptions. Thus, we impose the following weak continuity assumption.

DEFINITION 3.3-Lower Hemi-Continuity of $f$ : The dominance correspondence $f$ : $X \rightarrow X$ is lower hemi-continuous if for every sequence $\left(x^{k}\right)_{k \in \mathbb{N}}$ in $X$ such that $x^{k} \rightarrow x$ and for every $y \in f(x)$ there is a sequence $\left(y^{k}\right)_{k \in \mathbb{N}}$ in $X$ such that for all $k, y^{k} \in f\left(x^{k}\right)$ and $y^{k} \rightarrow y$.

This continuity assumption allows us to state the following uniqueness result.

THEOREM 3.4: Let $\Gamma$ be a social environment such that the corresponding dominance correspondence $f$ is lower hemi-continuous. Then $\Gamma$ has a unique MSS.

The continuity condition of Theorem 3.4 is trivially satisfied when the state space $X$ is finite. As such, for all applications with a finite state space, we have uniqueness of the MSS.

The dominance correspondence $f$ is defined in terms of the individual preference relations $\left(\succeq_{i}\right)_{i \in N}$ and the effectivity correspondence $E$. To ease the verification of lower hemi-continuity of $f$, we provide sufficient conditions on the primitives of a social environment.

As a first condition, we impose lower hemi-continuity of the effectivity correspondence $E$. Toward this end, consider, for every $S \in \mathcal{N}$, the correspondence $G_{S}: X \rightarrow X$ defined by

$$
G_{S}(x)=\{x\} \cup\{y \in X \mid S \in E(x, y)\}, \quad x \in X,
$$

which associates to every state $x \in X$ the union of $\{x\}$ and the set of states coalition $S$ can move to from $x$.

DEFINITION 3.5-Lower Hemi-Continuity of $E$ : The effectivity correspondence $E$ is lower hemi-continuous if for every coalition $S \in \mathcal{N}$ the correspondence $G_{S}: X \rightarrow X$ is lower hemi-continuous, that is, for every sequence $\left(x^{k}\right)_{k \in \mathbb{N}}$ in $X$ such that $x^{k} \rightarrow x$ and for every $y \in G_{S}(x)$ there is a sequence $\left(y^{k}\right)_{k \in \mathbb{N}}$ such that $y^{k} \in G_{S}\left(x^{k}\right)$ and $y^{k} \rightarrow y$.

Our second condition is continuity of the preferences. 
DEFINITION 3.6-Continuity of Preferences: The preference relation $\succeq_{i}$ of individual $i \in N$ is continuous if for any two sequences $\left(x^{k}\right)_{k \in \mathbb{N}}$ and $\left(y^{k}\right)_{k \in \mathbb{N}}$ in $X$ with $x^{k} \rightarrow x$ and $y^{k} \rightarrow y$ and, for every $k \in \mathbb{N}, x^{k} \succeq_{i} y^{k}$, it holds that $x \succeq_{i} y$.

The two conditions turn out to be sufficient for lower hemi-continuity of the dominance correspondence, thus by Theorem 3.4 for uniqueness of the MSS.

THEOREM 3.7: Let $\Gamma=\left(N,(X, d), E,\left(\succeq_{i}\right)_{i \in N}\right)$ be a social environment such that the effectivity correspondence $E$ is lower hemi-continuous and the preferences $\left(\succeq_{i}\right)_{i \in N}$ are continuous. Then the dominance correspondence $f$ is lower hemi-continuous and $\Gamma$ has a unique MSS.

In the online supplement, we construct a social environment which satisfies the conditions of Theorem 3.7, but has more than one weak dominance MSS.

\subsection{Closed Cycles and the Core}

In this subsection, we give two general results about the structure of a MSS. The first result relates the MSS to the union of all closed cycles. The second result characterizes the social environments for which the MSS is equal to the core.

DEFInITION 3.8-Closed Cycle: A closed cycle of a social environment $\Gamma$ is a set $C \subseteq X$ such that for every $x \in C$ it holds that $f^{\infty}(x)=C$.

Thus, a closed cycle is a subset of $X$ which is closed under the asymptotic dominance correspondence $f^{\infty}$. We denote the union of all closed cycles by CC, so CC contains all states that are part of some closed cycle. The following result characterizes the MSS for social environments with a finite state space as the union of all closed cycles and shows that this union is a subset of the MSS for social environments with an infinite state space.

THEOREM 3.9: Let $\Gamma=\left(N,(X, d), E,\left(\succeq_{i}\right)_{i \in N}\right)$ be a social environment and let $M$ be a $M S S$ of $\Gamma$. It holds that $\mathrm{CC} \subseteq M$. If the state space $X$ is finite, we have $\mathrm{CC}=M$.

A sink is a closed cycle which consists of only one state, that is, $f(x)=\{x\}$. The union of all sinks is called the core.

DEFINITION 3.10-Core: Let $\Gamma=\left(N,(X, d), E,\left(\succeq_{i}\right)_{i \in N}\right)$ be a social environment. The core $\mathrm{CO}$ of $\Gamma$ is given by

$$
\mathrm{CO}=\{x \in X \mid f(x)=\{x\}\} .
$$

It is well known that the core may be empty for some social environments. However, if it is not empty, then it is always contained in the MSS by the observation that a sink is a closed cycle which consists of one state and by virtue of Theorem 3.9.

COROLLARY 3.11: Let $\Gamma$ be a social environment and let $M$ be an MSS of $\Gamma$. Then we have $\mathrm{CO} \subseteq M$.

The next definition is inspired by the finite analogue for normal-form games as presented in Friedman and Mezzetti (2001). 
DEFINITION 3.12-Weak (Finite) Improvement Property: A social environment $\Gamma$ satisfies the weak finite improvement property if for each state $x \in X, f^{\mathbb{N}}(x)$ contains a sink and the weak improvement property if for each state $x \in X, f^{\infty}(x)$ contains a sink.

The following theorem provides a characterization for the MSS in social environments with the weak improvement property.

THEOREM 3.13: Let $\Gamma$ be a social environment with a lower hemi-continuous dominance correspondence $f$. An MSS of $\Gamma$ is equal to the core if and only if $\Gamma$ satisfies the weak improvement property.

It follows easily from the proof of Theorem 3.13 that the requirement of lower hemicontinuity of $f$ in Theorem 3.13 can be weakened to the requirement that $\mathrm{CO}$ is closed.

\subsection{The von Neumann-Morgenstern Stable Set}

The von Neumann-Morgenstern (vNM) stable set provides a solution concept for an environment consisting of a set of states $X$ and a dominance relation on this set (von Neumann and Morgenstern (1944)).

DEFINITION 3.14-vNM Stable Set: Let $\Gamma=\left(N,(X, d), E,\left(\succeq_{i}\right)_{i \in N}\right)$ be a social environment. The set $V \subseteq X$ is a $v N M$ stable set if it satisfies the following two conditions:

1. Internal stability: For all $x, y \in V$ such that $x \neq y$ it holds that $y \notin f(x)$.

2. External stability: For all $x \notin V, f(x) \cap V \neq \emptyset$.

Internal stability requires that no state in the set is dominated by another state in the set. External stability requires that every state outside the set should be dominated by a state in the set.

Our notion of asymptotic external stability has a similar flavor as the vNM notion of external stability. However, the vNM stable set looks at one-step dominations while our notion of asymptotic external stability uses asymptotic dominance, which can be seen as an infinite iteration of one-step dominations. In fact, extending the definition of the vNM stable set by allowing for a finite iteration of one-step dominations, that is, replacing $f$ by $f^{\mathbb{N}}$ in Definition 3.14, has also been advocated by several authors; see Harsanyi (1974), van Deemen (1991), Page and Wooders (2009), and Herings, Mauleon, and Vannetelbosch (2017).

On the other hand, our notion of deterrence of external deviations is quite different from the vNM notion of internal stability. While we allow that a state in the MSS is dominated by another state in the MSS, this is prohibited in the vNM stable set. Moreover, unlike our concept, in the vNM stable set it is allowed that a state in the set is dominated by a state outside the set.

In terms of predictions, first note that an MSS always exists and is unique under weak continuity assumptions, whereas the vNM stable set may not exist and if it exists may fail to be unique. If the vNM stable set exists, there are a few connections between the MSS and the vNM stable set. First of all, both sets contain the core. Second, the intersection between the vNM stable set and the MSS is nonempty as is stated in the next result.

THEOREM 3.15: Let $\Gamma=\left(N,(X, d), E,\left(\succeq_{i}\right)_{i \in N}\right)$ be a social environment for which a $v N M$ stable set $V$ exists. If $M$ is an MSS of $\Gamma$, then $M \cap V \neq \emptyset$. 
By Theorem 3.9, the MSS contains the union of all closed cycles. If the vNM stable set exists, one can show that it contains at least one state from every closed cycle.

It is easily verified that Theorem 3.15 remains true if the dominance correspondences $f^{\mathbb{N}}$ or $f^{\infty}$ are used in Definition 3.14.

\subsection{Dynamic Stochastic Processes}

Stochastic approaches have been frequently used in noncooperative settings like normal-form games. Sawa (2014) presents a general framework, which extends such a stochastic analysis to cooperative settings. In each period, one of the coalitions that can make a move is randomly selected and chooses one of its moves at random. The move is carried out with probability 1 if all members of the coalition are strictly better off. If no member is worse off, but at least one agent is indifferent, the move is carried out with a probability strictly between 0 and 1 . Otherwise, the move is not carried out. The resulting dynamic process can be thought of as a weak better-response dynamic. To establish a connection to the MSS, we consider a variation of the process in Sawa (2014) in which a coalition only moves with positive probability if all coalition members are strictly better off.

Consider a social environment $\Gamma=\left(N,(X, d), E,\left(\succeq_{i}\right)_{i \in N}\right)$ such that $X$ is finite. For states $x, y \in X$, let $Q(x, y)$ denote the transition probability from state $x$ to state $y$ and let $Q$ be the matrix of transition probabilities. We say that $Q$ is consistent with $f$ if for every $y \in f(x) \backslash\{x\}$ it holds that $Q(x, y)>0$ and for every $y \notin f(x)$ it holds that $Q(x, y)=0$. In particular, the state $x$ need not change even if the set $f(x) \backslash\{x\}$ is nonempty.

The next result presents an equivalence between the MSS and the set of recurrent states of the Markov chain $(X, Q)$.

THEOREM 3.16: Let $\Gamma=\left(N,(X, d), E,\left(\succeq_{i}\right)_{i \in N}\right)$ be a social environment with finite state space $X$, let $f$ be the corresponding dominance correspondence, and let $(X, Q)$ be a Markov chain such that $Q$ is consistent with $f$. Then the MSS of $\Gamma$ is equal to the set of recurrent states of $(X, Q)$.

For social environments with a finite number of states, Theorem 3.16 gives an equivalence between the set of recurrent states of a dynamic process that selects all better responses with positive probability and the MSS. Suppose we replace our dominance correspondence used in the definition of the MSS by requiring that $y$ indifference dominates $x$ if there exists a coalition $S \in E(x, y)$ such that for all $i \in S, y \succeq_{i} x$. Then the predictions of the alternative version of MSS are equivalent to the set of recurrent states in Sawa (2014) for the case of a finite state space.

The above results do not readily extend to settings where $X$ is infinite, as the set of recurrent states might be empty. In the online supplement, we discuss a stochastic approach to infinite environments based on irreducibility of the Markov chain and show that its predictions differ drastically from those of the MSS.

\section{APPLICATIONS}

In this section, we illustrate the generality of our setting and the useful common structure of our results by means of four specific models that have been studied extensively in the literature: coalition function form games, one-to-one matching models, models of network formation, and normal-form games. For each of these settings, we first specify 
the social environment, that is, the set of individuals $N$, the state space $(X, d)$, the effectivity correspondence $E$, and the preferences $\left(\succeq_{i}\right)_{i \in N}$. Subsequently, we discuss how the results from the previous section can be applied.

\subsection{Coalition Function Form Games}

A coalition function form game is defined by a tuple $(N, v)$, where $N$ is the set of players and $v: 2^{N} \rightarrow \mathbb{R}$ is a characteristic function that assigns to each coalition $S \subseteq N$ a number $v(S) \in \mathbb{R}$, called the coalitional value of $S$, with the usual convention that $v(\emptyset)=0$. A coalition structure is a partition $\pi$ of $N$. It describes how the grand coalition is divided into various sub-coalitions. The collection of all coalition structures, that is, the collection of partitions of $N$, is denoted by $\Pi$.

For coalition function form games, we define $X$ as the set of coalition structures $\Pi$ together with all individually rational payoff vectors that can be obtained by allocating the coalitional values among the members of the respective coalitions:

$$
X=\left\{(\pi, u) \in \Pi \times \mathbb{R}^{N} \mid \forall i \in N: u_{i} \geq v(\{i\}) \text { and } \forall S \in \pi: \sum_{i \in S} u_{i}=v(S)\right\} .
$$

Given a state $x \in X$, we denote by $\pi(x)$ the projection to its first component, that is, the coalition structure, and by $u(x)$ the projection to its second component, that is, the payoff vector, so we can write $x=(\pi(x), u(x))$. The restriction of the payoff vector $u(x)$ to the members in coalition $S$ is denoted by $u_{S}(x)$. The set $X$ is nonempty since it always contains the state where $N$ is partitioned into singletons and each player $i \in N$ receives the payoff $v(\{i\})$.

For $x, y \in X$, we define $d(x, y)=\mathbb{1}_{\{\pi(x) \neq \pi(y)\}}+\|u(x)-u(y)\|_{\infty}$, where $\mathbb{1}$ is the indicator function and $\|\cdot\|_{\infty}$ is the infinity norm. It is easily seen that $(X, d)$ is compact. We define preferences $\succeq_{i}$ over the state space $X$ by setting $x \succeq_{i} y$ if and only if $u_{i}(x) \geq u_{i}(y)$.

For each ordered pair of states $(x, y)$, the effectivity correspondence $E(x, y)$ specifies which coalitions can change state $x$ into state $y$. As an example that imposes some reasonable structure on the effectivity correspondence, we provide a brief outline of the notion of coalitional sovereignty (Konishi and Ray (2003), Kóczy and Lauwers (2004), Ray and Vohra (2014, 2015), Herings, Mauleon, and Vannetelbosch (2017)).

When a coalition of players $S$-called the leaving players-decides to leave their old coalitions to create a new group, the state changes to a new state $y$ characterized by a new coalition structure $\pi(y)$ and a new payoff vector $u(y)$. The collection of coalitions of $\pi(x)$ that are unaffected by this change is denoted by $\mathcal{U}(x, S)$ and the set of all players in this group by $U(x, S)$. Formally, we have $\mathcal{U}(x, S)=\{T \in \pi(x) \mid S \cap T=\emptyset\}$ and $U(x, S)=$ $\bigcup_{T \in \mathcal{U}(x, S)} T$.

This notation helps us in defining coalitional sovereignty.

DEFINITION 4.1-Coalitional Sovereignty: An effectivity correspondence $E$ satisfies coalitional sovereignty if the following two conditions hold:

(1) Noninterference: For every $x, y \in X$, if $S \in E(x, y)$ and $T \in \mathcal{U}(x, S)$, then $S \in \pi(y)$, $T \in \pi(y)$, and $u_{T}(x)=u_{T}(y)$.

(2) Full support: For every $x \in X$, for every $S \in \mathcal{N}$, and for every $u \in \mathbb{R}^{S}$ such that for all $i \in S: u_{i} \geq v(\{i\})$ and $\sum_{i \in S} u_{i}=v(S)$, there is a state $y \in X$ such that $S \in E(x, y)$ and $u_{S}(y)=u$. 
Intuitively, noninterference requires that if a coalition $S$ induces a change from a state $x$ to a state $y$, then the unaffected coalitions in $\mathcal{U}(x, S)$ are still part of the new coalition structure $\pi(y)$ and every unaffected player $i \in U(x, S)$ keeps his old payoff, that is, $u_{i}(x)=u_{i}(y)$. Full support requires that every coalition $S$ has the opportunity to move to a new state where it has the freedom to redistribute its worth $v(S)$ at will.

Coalitional sovereignty does not fully specify the effectivity correspondence $E$. In particular, it does not specify the payoffs and coalition structure of players that are neither part of the leaving coalition $S$ nor part of the unaffected players $U(x, S)$, that is, players in the set $N \backslash(S \cup U(x, S))$. We call these players residual players. Indeed, one of the more controversial issues is to what extent the leaving players have the power to influence the coalition structure and payoffs of these residual players; see Shubik (1962), Hart and Kurz (1983), Konishi and Ray (2003), and Ray and Vohra (2014) for related discussions and alternative viewpoints. One frequently used specification is the $\gamma$-model (Hart and Kurz (1983)). The $\gamma$-model prescribes that the residual players are divided into singletons. This assumption is justified by the viewpoint that a coalition is only maintained if there is unanimous agreement among its members. In our setting, the $\gamma$-model imposes the following restriction on the effectivity correspondence.

DEFINITION 4.2- $\gamma$-Model: The effectivity correspondence $E$ is induced by the $\gamma$ model if it satisfies coalitional sovereignty and

(3) For every $x, y \in X$, for every $S \in E(x, y)$, if $i \in N \backslash(S \cup U(x, S))$, then $\{i\} \in \pi(y)$.

The $\gamma$-model associates a unique social environment to each coalition function form game.

One of the most prominent set-valued solution concepts for coalition function form games is the coalition structure core.

DEFINITION 4.3-Coalition Structure Core: Let $(N, v)$ be a coalition function form game and $\Gamma=\left(N,(X, d), E,\left(\succeq_{i}\right)_{i \in N}\right)$ be the social environment induced by the $\gamma$-model. The coalition structure core of $(N, v)$ is the set of states $x \in X$ such that, for every coalition $S \in \mathcal{N}, \sum_{i \in S} u_{i}(x) \geq v(S)$.

In words, the coalition structure core gives to the members of each coalition at least the payoff they can obtain by forming that coalition.

Kóczy and Lauwers (2004) defined the coalition structure core to be accessible if from any initial state there is a finite sequence of states ending with an element of the coalition structure core and each element in that sequence outsider independently dominates the previous element. Accessibility of the coalition structure core thus corresponds to iterated external stability of the coalition structure core with respect to outsider independent domination. The notion of outsider independent domination differs from our notion of a myopic improvement in the $\gamma$-model in two ways. First, it gives complete freedom to the treatment of residual players after a move has taken place. Second, improvements for the members of the coalition that moves are not necessarily strict improvements. In the online supplement, we give an example where under the requirement of strict improvements of all members involved in a move, as in our dominance correspondence $f$, the coalition structure core does not satisfy iterated external stability. However, the definition of an MSS uses asymptotic external stability rather than iterated external stability.

By Theorem 3.1, there exists at least one MSS. Theorem 4.4 shows the MSS to be unique and to coincide with the coalition structure core whenever the coalition structure core is nonempty. 
THEOREM 4.4: Let $(N, v)$ be a coalition function form game and $\Gamma$ the social environment induced by the $\gamma$-model. Then $\Gamma$ has a unique MSS. If the coalition structure core $Y$ of $(N, v)$ is nonempty, then the MSS of $\Gamma$ is equal to $Y$.

Theorem 4.4 covers coalition function form games with a nonempty coalition structure core. We now study the predictions of MSS when the coalition structure core is empty. To do so, we analyze the class of proper simple games which play an important role in the analysis of political institutions. These are coalition function form games such that $v(N)=1$, for every $S \subseteq N$ it holds that $v(S) \in\{0,1\}$, and $v(S)=1$ implies $v(N \backslash S)=0$. Moreover, for $S, T \subseteq N$ such that $S \subseteq T, v(S)=1$ implies $v(T)=1$. The collection of winning coalitions is denoted by $\mathcal{W}=\{S \subseteq N \mid v(S)=1\}$. A veto player is a player that belongs to every winning coalition and the set of veto players is given by $S^{*}=\bigcap_{S \in \mathcal{W}} S$.

It is well known that the coalition structure core of a proper simple game is nonempty if and only if there are veto players, so $S^{*} \neq \emptyset$. In that case, the coalition structure core, and by Theorem 4.4 the MSS, is given by the states $x \in X$ such that $\pi(x)$ has an element containing $S^{*}$ and $\sum_{i \in S^{*}} u_{i}(x)=1$.

Let us now consider proper simple games with an empty core. Define the subset $F(X)$ of $X$ as the set of states such that its partition contains a winning coalition different from the grand coalition and all other elements of the partition are singletons:

$$
F(X)=\{x \in X \mid \pi(x) \cap(\mathcal{W} \backslash\{N\}) \neq \emptyset \text { and } \forall S \in \pi(x) \backslash \mathcal{W},|S|=1\} .
$$

If $x \in F(X)$, then the payoff vector $u(x)$ distributes one unit of surplus to the players, each player has a nonnegative payoff, and at least one player has a payoff of zero. The payoff vector therefore belongs to a facet of the unit simplex.

THEOREM 4.5: Let $(N, v)$ be a coalition function form game such that $v$ is a proper simple game with an empty core and let $\Gamma=\left(N,(X, d), E,\left(\succeq_{i}\right)_{i \in N}\right)$ be the social environment induced by the $\gamma$-model. Then the MSS of $\Gamma$ is unique and equal to $F(X)$.

Each state in the MSS of the $\gamma$-model for simple games with an empty core contains a winning coalition that is a proper subset of the grand coalition. All other coalitions fall apart into singletons. In the online supplement, we consider the $\delta$-model as suggested by Hart and Kurz (1983) where residual players remain together. In payoff terms, the predictions of the $\gamma$-model and the $\delta$-model are shown to coincide, though in the $\delta$-model the non-winning coalitions need not be singletons. In the online supplement, we also compare the predictions of the MSS with the vNM stable set for three-player simple games with either one, two, or three winning two-player coalitions. In the first case, both concepts coincide. In the second case, there are two vNM stable sets each with a continuum of states and containing the finite MSS as a proper subset. In the third case, there are four vNM stable sets and their union contains the MSS as a proper subset.

\subsection{Matching}

As a second application, we consider the one-to-one matching model of Gale and Shapley (1962) and the one-to-one matching model of Knuth (1976). In the online supplement, we also discuss the housing matching model of Shapley and Scarf (1974).

Gale and Shapley (1962) introduced a one-to-one matching model for a finite set $N$ of individuals, partitioned in the two exhaustive subgroups, men $M$ and women $W$. The model can be described by a tuple $\left(M, W,\left(P_{m}\right)_{m \in M},\left(P_{w}\right)_{w \in W}\right)$ of individuals and their 
preference relations. A matching is a function $\mu: M \cup W \rightarrow M \cup W$ satisfying the following properties:

1. For every man $m \in M, \mu(m) \in W \cup\{m\}$.

2. For every woman $w \in W, \mu(w) \in M \cup\{w\}$.

3. For all men $m \in M$ and women $w \in W, \mu(m)=w$ if and only if $\mu(w)=m$.

In this setting, our state space $X$ consists of all possible matchings $\mu$. Since $X$ is finite, we can endow it with the discrete metric $d\left(\mu, \mu^{\prime}\right)=\mathbb{1}_{\left\{\mu \neq \mu^{\prime}\right\}}$. Each man $m \in M$ has a complete and transitive strict preference relation $P_{m}$ over the set $W \cup\{m\}$ and each woman $w \in W$ has a complete and transitive strict preference relation $P_{w}$ over the set $M \cup\{w\}$. The preferences of the individuals $\left(\succeq_{i}\right)_{i \in M \cup W}$ over the set $X$ are induced by their preferences over their match, that is, for all $m \in M$ it holds that $\mu \succ_{m} \mu^{\prime}$ if and only if $\mu(m) P_{m} \mu^{\prime}(m)$ and for all $w \in W$ it holds that $\mu \succ_{w} \mu^{\prime}$ if and only if $\mu(w) P_{w} \mu^{\prime}(w)$.

The formulation of the effectivity correspondence allows us to study the consequences of different hypothesis on the matching process. We introduce two common assumptions from the literature on matching. First, every man or woman is allowed to break the link with the current partner, in which case this man or woman and the former partner become single:

(1) For all $i \in N$ and $\mu \in X$ with $\mu(i) \neq i$, we have $\{i\} \in E\left(\mu, \mu^{\prime}\right)$ where $\mu^{\prime} \in X$ is such that

(i) $\mu^{\prime}(i)=i$,

(ii) $\mu^{\prime}(\mu(i))=\mu(i)$,

(iii) for every $j \in N \backslash\{i, \mu(i)\}, \mu^{\prime}(j)=\mu(j)$.

The second assumption is that any man and woman that are currently not matched to each other can deviate by creating a link and thereby leaving their former partners single:

(2) For all $m^{\prime} \in M, w^{\prime} \in W$, and $\mu \in X$ with $\mu\left(m^{\prime}\right) \neq w^{\prime}$, we have that $\left\{m^{\prime}, w^{\prime}\right\} \in$ $E\left(\mu, \mu^{\prime}\right)$, where $\mu^{\prime} \in X$ is such that

(i) $\mu^{\prime}\left(m^{\prime}\right)=w^{\prime}$,

(ii) $\mu\left(m^{\prime}\right) \in W$ implies $\mu^{\prime}\left(\mu\left(m^{\prime}\right)\right)=\mu\left(m^{\prime}\right)$,

(iii) $\mu\left(w^{\prime}\right) \in M$ implies $\mu^{\prime}\left(\mu\left(w^{\prime}\right)\right)=\mu\left(w^{\prime}\right)$,

(iv) for every $j \in N \backslash\left\{m^{\prime}, w^{\prime}, \mu\left(m^{\prime}\right), \mu\left(w^{\prime}\right)\right\}, \mu^{\prime}(j)=\mu(j)$.

Observe that these two conditions are in line with the $\gamma$-model of coalitional sovereignty. This completes the description of the effectivity correspondence and thereby of the social environment of the model by Gale and Shapley (1962).

Let $\left(M, W,\left(P_{m}\right)_{m \in M},\left(P_{w}\right)_{w \in W}\right)$ be a matching problem. A matching $\mu$ is said to be stable if for every $i \in M \cup W$ it does not hold that $i P_{i} \mu(i)$ and if for every pair $(m, w) \in M \times W$ it does not hold that $w P_{m} \mu(m)$ and $m P_{w} \mu(w)$. It can easily be shown that a matching is stable if and only if it is in the core of the social environment $\Gamma$.

In their seminal contribution, Gale and Shapley (1962) showed the existence of a stable matching. The following result of Roth and Vande Vate (1990) is helpful in determining the relation between the set of stable matchings and the MSS.

THEOREM 4.6-Roth and Vande Vate (1990): For every matching $\mu \in X$, there is a stable matching $\mu^{\prime}$ such that $\mu^{\prime} \in f^{\mathbb{N}}(\mu)$.

Since the set of states is finite in this application, it holds that $f^{\mathbb{N}}(\mu)=f^{\infty}(\mu)$. Recalling Definition 3.12, the result of Roth and Vande Vate (1990) means that $\Gamma$ satisfies the weak improvement property. For finite settings, $f$ is always lower hemi-continuous. Thus, by Theorem 3.13, the MSS of the social environment induced by the one-to-one matching model coincides with the set of stable matchings, which is the statement of the following corollary. 
COROLLARY 4.7: Let $\left(M, W,\left(P_{m}\right)_{m \in M},\left(P_{w}\right)_{w \in W}\right)$ be a matching problem and let $\Gamma$ be the induced social environment. The MSS of $\Gamma$ is unique and equal to the set of stable matchings.

An alternative one-to-one matching model is due to Knuth (1976). This model differs from the model of Gale and Shapley (1962) in that no individual is allowed to be single. Therefore, it requires the number of men to be equal to the number of women. If a blocking pair forms, the deserted partners are matched together. The primitives of the matching model are given by a tuple $\left(M, W,\left(P_{m}\right)_{m \in M},\left(P_{w}\right)_{w \in W}\right)$ with $|M|=|W|$. A matching is a function $\mu: M \cup W \rightarrow M \cup W$ satisfying the following properties:

1. For every man, $m \in M, \mu(m) \in W$.

2. For every women, $w \in W, \mu(w) \in M$.

3. For all men $m \in M$ and women, $w \in W, \mu(m)=w$ if and only if $\mu(w)=m$.

The state space $X$ consists of all matchings $\mu$ satisfying the above three properties and is endowed with the discrete metric $d\left(\mu, \mu^{\prime}\right)=\mathbb{1}_{\left\{\mu \neq \mu^{\prime}\right\}}$. The preferences of the individuals $\left(\succeq_{i}\right)_{i \in M \cup W}$ over the set $X$ are induced by their preferences over their match, that is, for all $m \in M$ it holds that $\mu \succ_{m} \mu^{\prime}$ if and only if $\mu(m) P_{m} \mu^{\prime}(m)$ and for all $w \in W$ it holds that $\mu \succ_{w} \mu^{\prime}$ if and only if $\mu(w) P_{w} \mu^{\prime}(w)$. If a man and woman create a new link, the effectivity correspondence also requires a link between their deserted partners. Formally, for all $m^{\prime} \in M, w^{\prime} \in W$, and $\mu \in X$ with $\mu\left(m^{\prime}\right) \neq w^{\prime}$, we have that $\left\{m^{\prime}, w^{\prime}\right\} \in E\left(\mu, \mu^{\prime}\right)$, where $\mu^{\prime} \in X$ is such that

1. $\mu^{\prime}\left(m^{\prime}\right)=w^{\prime}$,

2. $\mu^{\prime}\left(\mu\left(w^{\prime}\right)\right)=\mu\left(m^{\prime}\right)$,

3. for every $j \in N \backslash\left\{m^{\prime}, w^{\prime}, \mu\left(m^{\prime}\right), \mu\left(w^{\prime}\right)\right\}, \mu^{\prime}(j)=\mu(j)$.

This completes the definition of the effectivity correspondence and thereby of the social environment.

The core of the social environment induced by the Knuth (1976) model is nonempty. Moreover, as shown by Tamura (1993), when there are at least four women, there are preferences and a matching $\mu \in X$ such that $f^{\infty}(\mu)$ does not contain a stable matching. In these cases, the MSS contains matchings outside the core and can thus be rather large. We present such an example in the online supplement and also compare the MSS to the vNM stable set for that example.

To obtain an intuition which states outside the core are part of a MSS, recall that, by Theorem 3.9, the MSS coincides with the union of all closed cycles. Thus, if the MSS contains states outside the core, these states are part of a closed cycle with more than one element. In such a cycle, agents myopically form new matches and eventually come back to the initial match. These additional states are included in the MSS due to two restrictions on the agents. First, agents are myopic, and thus only consider deviations which result in an immediate gain. Second, agents are additionally restricted by the effectivity correspondence which only allows for pairwise deviations.

\subsection{Network Formation}

As a third application, we look at the model of network formation by Jackson and Wolinsky (1996). A network is given by a tuple $g=(N, \mathcal{E})$, where the nodes $N$ are the players of the network and $\mathcal{E}$ is the set of undirected edges of the network. An undirected edge is represented as a set of two distinct players. Two players $i, j \in N$ are linked in $g$ if and only if $\{i, j\} \in \mathcal{E}$. We abuse notation and write $i j \in g$ if $i$ and $j$ are linked in the network $g$. The set of all networks with node set $N$ is denoted by $\mathbb{G}$. A value function for player $i$ is a function $v_{i}: \mathbb{G} \rightarrow \mathbb{R}$ that associates payoffs for player $i$ for each network in $\mathbb{G}$. A network problem is thus given by $\left(N, \mathbb{G},\left(v_{i}\right)_{i \in N}\right)$. 
We identify $X$ with the set $\mathbb{G}$ of all possible networks on $N$ and endow it with the discrete metric $d\left(g, g^{\prime}\right)=\mathbb{1}_{\left\{g \neq g^{\prime}\right\}}$. Every player $i \in N$ has a preference relation $\succeq_{i}$ over the set $X$ of all possible networks defined by $g \succeq_{i} g^{\prime}$ if and only if $v_{i}(g) \geq v_{i}\left(g^{\prime}\right)$. Let $g+i j$ be the network obtained from network $g$ by adding the link $i j$ to $g$ and let $g-i j$ be the network obtained by deleting link $i j$ from $g$.

We follow Jackson and Wolinsky (1996) by considering deviations by coalitions of size one or two and by assuming link deletion to be one-sided and link addition to be twosided. One-sided link deletion allows every player to delete one of his links:

(1) For all players $i \in N$, all networks $g \in X$, and all links $i j \in g,\{i\} \in E(g, g-i j)$. Two-sided link addition allows any two players that are currently not linked to change the network by forming a link between themselves:

(2) For all players $i, j \in N$, all networks $g \in X$ with $i j \notin g$, we have $\{i, j\} \in E(g, g+i j)$. This completes the description of our social environment for the network formation model. It is straightforward to adjust the effectivity correspondence to incorporate models of network formation where more than one link at a time can be changed by coalitions of arbitrary size (Dutta and Mutuswami (1997), Jackson and van den Nouweland (2005)) or where link formation is one-sided (Bala and Goyal (2000)) into our framework. We refer to Page and Wooders (2009) for a more extensive discussion of alternative rules of network formation.

A network $g$ is said to be pairwise stable (Jackson and Wolinsky (1996)) if for every $i j \in g$ it holds that $v_{i}(g-i j) \leq v_{i}(g)$ and $v_{j}(g-i j) \leq v_{j}(g)$ and for every $i j \notin g$ it holds that $v_{i}(g+i j)>v_{i}(g)$ implies $v_{j}(g+i j) \leq v_{j}(g){ }^{2}$ It is not hard to show that a network is pairwise stable if and only if it is in the core of the social environment $\Gamma$ as defined in Definition 3.10.

Corollary 3.11 shows that any pairwise stable network is in the myopic stable set. However, it is not necessarily the case that the MSS only contains the pairwise stable networks.

Consider the binary relation $R$ on $X$ defined by $g R g^{\prime}$ if $g \in f^{\mathbb{N}}\left(g^{\prime}\right)$, that is, $g$ can be reached from $g^{\prime}$ by a finite number of dominations. Let $I$ be the symmetric part of $R$, that is, $g I g^{\prime}$ if and only if $g R g^{\prime}$ and $g^{\prime} R g$. Consider the set of equivalence classes $\mathbb{E}$ induced by $I$. Denote the equivalence class of network $g$ by $[g]$, that is, $g^{\prime} \in[g]$ if and only if $g^{\prime} I g$. For two distinct equivalence classes $[g]$ and $\left[g^{\prime}\right]$, write $[g] P\left[g^{\prime}\right]$ if $g R g^{\prime}$. It is easy to see that $[g] P\left[g^{\prime}\right]$ if and only if $g R g^{\prime}$ and not $g R g^{\prime}$.

Let $V$ be the collection of maximal elements of $(\mathbb{E}, P)$, that is, $[g] \in V$ if there is no $\left[g^{\prime}\right] \in \mathbb{E}$ such that $\left[g^{\prime}\right] P[g]$. Since an element of $V$ simply represents a closed cycle as defined in Definition 3.8, the following result follows from Theorem 3.9.

COROLLARY 4.8: Let $\left(N, \mathbb{G},\left(v_{i}\right)_{i \in N}\right)$ be a network problem and let $\Gamma$ be the induced social environment. A network $g$ belongs to the unique MSS $M$ of $\Gamma$ if and only if the equivalence class [ $g]$ belongs to $V$, that is, $M=\{g \in X \mid[g] \in V\}$.

Herings, Mauleon, and Vannetelbosch (2009) defined the pairwise myopically stable sets for network problems using pairwise stability as defined in Section 2 of Jackson and Wolinsky (1996). It is not hard to see that the MSS for social environments $\Gamma$ coincides with the pairwise myopically stable set for generic network problems. For such network

\footnotetext{
${ }^{2}$ Pairwise stability as defined in Section 2 of Jackson and Wolinsky (1996) is somewhat stronger and also requires that there is no $i j \notin g$ such that $v_{i}(g+i j)>v_{i}(g)$ and $v_{j}(g+i j)=v_{j}(g)$. The weaker notion used here is discussed as an alternative in Section 5 of Jackson and Wolinsky (1996) and is also widely used in the literature. For generic network problems, there are no indifferences, so the two definitions are equivalent.
} 
problems, Corollary 4.8 is therefore equivalent to Theorem 1 of Herings, Mauleon, and Vannetelbosch (2009) that characterizes the pairwise myopically stable set as the union of closed cycles. In their paper, a closed cycle is defined in the sense of Jackson and Watts (2002) for network problems. The notion of closed cycle in Definition 3.8 is the appropriate generalization to social environments.

\subsection{Normal-Form Games}

As a final application, we consider normal-form games. We consider social environments where players are restricted to the use of pure strategies. The online supplement contains a formalization of the mixed extension with several results for that case.

A normal-form game $G=\left(N,\left(\left(\Sigma_{i}, d_{i}\right), u_{i}\right)_{i \in N}\right)$ consists of a set of players $N$ and for each player $i \in N$ a nonempty and compact metric space $\left(\Sigma_{i}, d_{i}\right)$ of pure strategies and a utility function $u_{i}: \Sigma \rightarrow \mathbb{R}$ over the set of strategy profiles $\Sigma=\prod_{i \in N} \Sigma_{i}$. A typical element of $\Sigma$ is denoted by $s$.

For the corresponding social environment $\Gamma=\left(N,(X, d), E,\left(\succeq_{i}\right)_{i \in N}\right)$, we equate the state space $X$ with the set of strategy profiles $\Sigma$ and endow it with the product metric $d\left(s, s^{\prime}\right)=\sum_{i \in N} d_{i}\left(s_{i}, s_{i}^{\prime}\right)$. The preferences $\left(\succeq_{i}\right)_{i \in N}$ are such that $s \succeq_{i} s^{\prime}$ if and only if $u_{i}(s) \geq$ $u_{i}\left(s^{\prime}\right)$.

We write $\left(s_{S}, s_{-S}\right)$ for the strategy profile where $s_{S}$ is the list of strategies of players in coalition $S \in \mathcal{N}$ and $s_{-S}$ is the list of strategies of all other players, that is, $s_{S}=\left(s_{j}\right)_{j \in S}$ and $s_{-S}=\left(s_{j}\right)_{j \in N \backslash S}$. With a slight abuse of notation, we write $s_{i}$ and $s_{-i}=\left(s_{j}\right)_{j \in N \backslash\{i\}}$ for single-player coalitions $S=\{i\}$.

It remains to specify the effectivity correspondence $E$ of the social environment $\Gamma$. By allowing for all coalitional deviations, we describe coalitional normal-form games. Formally, for a coalition $S \in \mathcal{N}$, the effectivity correspondence is such that $S \in E\left(s, s^{\prime}\right)$ if and only if $s_{-S}=s_{-S}^{\prime}$. For this effectivity correspondence, a strategy profile $s \in \Sigma$ is a strong Nash equilibrium (Aumann (1959)) if and only if it is in the core of the social environment $\Gamma=\left(N,(X, d), E,\left(\succeq_{i}\right)_{i \in N}\right)$. Thus, any strong Nash equilibrium must be in the MSS of the associated social environment.

Instead of the coalitional approach, we will henceforth restrict attention to the more frequently analyzed case of a noncooperative game. In this case, the effectivity correspondence $E$ only allows singletons to move. Formally, we have that $S \in E\left(s, s^{\prime}\right)$ if and only if $s_{-S}=s_{-S}^{\prime}$ and $|S|=1$. A non-cooperative normal-form game $G=\left(N,\left(\left(\Sigma_{i}, d_{i}\right), u_{i}\right)_{i \in N}\right)$ then induces a social environment $\Gamma=\left(N,(X, d), E,\left(\succeq_{i}\right)_{i \in N}\right)$ that is identical to the social environment defined for normal-form games with coalitional moves, except that the effectivity correspondence $E$ only allows for coalitions of size one.

A strategy profile $s \in \Sigma$ is said to be a pure strategy Nash equilibrium of the game $G$ if for every $i \in N$ and for every $s_{i}^{\prime} \in \Sigma_{i}$ it holds that $u_{i}(s) \geq u_{i}\left(s_{i}^{\prime}, s_{-i}\right)$. Note that a strategy profile is a pure strategy Nash equilibrium if and only if it is in the core of the social environment $\Gamma$. Corollary 3.11 then shows that every pure strategy Nash equilibrium belongs to every MSS.

In the next step, we define pseudo-potential games (Dubey, Haimanko, and Zapechelnyuk (2006)) and show that the MSS coincides with the set of pure-strategy Nash equilibria for this class of games.

DEFINITION 4.9-Pseudo-Potential Game: The game $G=\left(N,\left(\left(\Sigma_{i}, d_{i}\right), u_{i}\right)_{i \in N}\right)$ is a pseudo-potential game if there exists a continuous function $P: \Sigma \rightarrow \mathbb{R}$ such that, for all $i \in N$, for all $s \in \Sigma, \arg \max _{s_{i} \in \Sigma_{i}} u_{i}\left(s_{i}, s_{-i}\right) \supseteq \arg \max _{s_{i} \in \Sigma_{i}} P\left(s_{i}, s_{-i}\right)$. 
Pseudo-potential games generalize ordinal potential games (Monderer and Shapley (1996)) and best-response potential games (Voorneveld (2000)). Moreover, Dubey, Haimanko, and Zapechelnyuk (2006) showed that the class of pseudo-potential games contains games of strategic complements or substitutes with aggregation such as Cournot oligopoly games. Jensen (2010) extended this result to generalized quasi-aggregative games. ${ }^{3}$

THEOREM 4.10: Let $G=\left(N,\left(\left(\Sigma_{i}, d_{i}\right), u_{i}\right)_{i \in N}\right)$ be a pseudo-potential game and let $\Gamma$ be the induced social environment. If the utility functions $\left(u_{i}\right)_{i \in N}$ are continuous, then the MSS of $\Gamma$ coincides with the set of pure strategy Nash equilibria of $G$.

For finite supermodular games, Friedman and Mezzetti (2001) establish the weak finite improvement property which implies the weak improvement property. Thus, the equivalence between the set of pure strategy Nash equilibria and the MSS also extends to this class of games.

\section{CONCLUSION}

The myopic stable set provides a solution concept for a wide variety of social environments. As we have shown, the setting encompasses coalition function form games, models of network formation, matching models, and noncooperative games. These environments have been chosen based on their prominence in the literature but are by no means exhaustive. In particular, promising environments for future research on the myopic stable set include exchange processes in general equilibrium models and many-to-many matching models with transfers.

The following three features boost the appeal of the myopic stable set as a solution concept. First, the myopic stable set unifies standard solution concepts in many social environments. For instance, it coincides with the coalition structure core in coalition function form games (Kóczy and Lauwers (2004)) if the coalition structure core is nonempty, the set of stable matchings in the one-to-one matching model of Gale and Shapley (1962), the set consisting of pairwise stable networks and closed cycles of networks (Jackson and Watts (2002), and the set of pure strategy Nash equilibria in finite supermodular games (Topkis (1979) and Milgrom and Roberts (1990) and pseudo-potential games (Dubey, Haimanko, and Zapechelnyuk (2006).

Second, our solution concept exists for any social environment and—under weak continuity assumptions - provides a unique set-valued prediction. This differs from well-known concepts in the literature which fail to satisfy these properties even in social environments with more structure.

Third, in important classes of problems, the MSS gives sharp predictions. For instance, for matching markets empirical findings starting with Roth and Peranson (1999) suggested that the core is small and Ashlagi, Kanoria, and Leshno (2017) provided theoretical arguments for why this is the case. The equivalence between the core of matching problems and the MSS then implies that MSS has significant predictive power. Other examples are the equivalence between MSS and the set of pure Nash equilibria pseudo-potential games and finite supermodular games.

\footnotetext{
${ }^{3}$ Generalized quasi-aggregative games include aggregative games (Selten (1970)). For a subclass of aggregative games, the equivalence result in Theorem 4.10 can be obtained from Dindoš and Mezzetti (2006).
} 
At the same time, there are cases where the MSS may be large. For example, when the MSS contains states that do not belong to the core as in the matching model by Knuth (1976) as discussed in Section 4.2. Intuitively, the combination of myopic behavior and a restrictive effectivity correspondence may result in cycling and hence, a large MSS. This reflects the trade-off between a general solution concept for which existence and nonemptiness is guaranteed, like the MSS, and a clear prediction for every class of social environments. The investigation of refinements of the MSS in such cases is a natural direction for future research.

\section{APPENDIX: PROOFS}

Proof OF THEOREM 3.1: First, observe that the set of states $X$ is a QMSS. Indeed, since it is compact, it is closed and it trivially satisfies deterrence of external deviations and asymptotic external stability.

Let $\mathcal{Z}$ be the collection of all sets of states that are a QMSS. Notice that $\mathcal{Z}$ is nonempty as $X \in \mathcal{Z}$. A set $Z^{\prime} \in \mathcal{Z}$ is a maximal element in the partially ordered set $(\mathcal{Z}, \supseteq)$ if for all $Z \in \mathcal{Z}$ with $Z^{\prime} \supseteq Z$, we have $Z=Z^{\prime}$. We will use Zorn's lemma to show the existence of a maximal element in the partially ordered set $(\mathcal{Z}, \supseteq)$.

Let $\mathcal{S}$ be a chain in $\mathcal{Z}$, that is, $(\mathcal{S}, \supseteq)$ is a totally ordered subset of $(\mathcal{Z}, \supseteq)$. Let $I$ be an index set for the sets in $\mathcal{S}$, that is, $\mathcal{S}=\left\{Z^{\alpha} \mid \alpha \in I\right\}$. Let $\triangleright$ be the order on $I$ that is induced by the order on $\mathcal{S}$, that is, $\beta \triangleright \alpha$ if and only if $Z^{\alpha} \supseteq Z^{\beta}$. In order to apply Zorn's lemma, we have to show that $\mathcal{S}$ has an upper bound in $\mathcal{Z}$. Let $M=\bigcap_{\alpha \in I} Z^{\alpha}$. Clearly, $M$ is an upper bound of $\mathcal{S}$. We proceed by showing that $M \in \mathcal{Z}$, that is, $M$ is a QMSS. First of all, observe that $M$ is closed as it is defined as the intersection of a collection of closed sets. We need to show that it satisfies deterrence of external deviations and asymptotic external stability.

Deterrence of external deviations: Let $x \in M$ and $y \notin M$ be given. Then there is $\alpha \in I$ such that $y \notin Z^{\alpha}$, since otherwise $y \in Z^{\alpha}$ for all $\alpha \in I$, which means that $y \in M$. Since $x \in Z^{\alpha}$ and $Z^{\alpha}$ satisfies deterrence of external deviations, we obtain $y \notin f(x)$ as was to be shown.

Asymptotic external stability: Consider some $y \notin M$. Then there is $\alpha \in I$ such that $y \notin Z^{\alpha}$. As $\mathcal{S}$ is a chain, it follows that for all $\beta \triangleright \alpha$ we have $y \notin Z^{\beta}$.

For every $\beta \triangleright \alpha$, there is $x^{\beta} \in Z^{\beta}$ such that $x^{\beta} \in f^{\infty}(y)$, since $Z^{\beta}$ satisfies asymptotic external stability. This defines a net $\left(x^{\beta}\right)_{\beta \triangleright \alpha}$. Given that $X$ is compact, it follows by Theorem 2.31 of Aliprantis and Border (2006) that this net has a convergent subnet, say $\left(x^{\beta^{\prime}}\right)_{\beta^{\prime} \in I^{\prime}}$, where $I^{\prime} \subseteq I$ is such that for all $\beta \in I$ there is a $\beta^{\prime} \in I^{\prime}$ such that $\beta^{\prime} \triangleright \beta$. Let $\bar{x}$ be the limit of this convergent subnet. We split the remaining part of the proof in two steps. First, we show that $\bar{x} \in M$. Second, we show that $\bar{x} \in f^{\infty}(y)$.

Step 1: $\bar{x} \in M$ : Toward a contradiction, suppose that $\bar{x} \notin M$. Then there exists $\gamma \in I$ such that $\bar{x} \notin Z^{\gamma}$. In particular, given that $Z^{\gamma}$ is a closed set, there is $\varepsilon>0$ such that $B_{\varepsilon}(\bar{x}) \cap Z^{\gamma}=\emptyset$. Since $\mathcal{S}$ is a chain, we have that $B_{\varepsilon}(\bar{x}) \cap Z^{\delta}=\emptyset$ for all $\delta \triangleright \gamma$. Since $\bar{x}$ is the limit of the subnet $\left(x^{\beta^{\prime}}\right)_{\beta^{\prime} \in I^{\prime}}$, there is $\gamma^{\prime} \in I^{\prime}$ such that $\gamma^{\prime} \triangleright \gamma$ and $x^{\gamma^{\prime}} \in B_{\varepsilon}(\bar{x})$. Then we have $x^{\gamma^{\prime}} \in Z^{\gamma^{\prime}}, x^{\gamma^{\prime}} \in B_{\varepsilon}(\bar{x})$, and $B_{\varepsilon}(\bar{x}) \cap Z^{\gamma^{\prime}}=\emptyset$, a contradiction. We conclude that $\bar{x} \in M$.

Step 2: $\bar{x} \in f^{\infty}(y)$ : We need to show that for every $\varepsilon>0$ there is $k \in \mathbb{N}$ and $x \in f^{k}(y)$ such that $x \in B_{\varepsilon}(\bar{x})$.

Let some $\varepsilon>0$ be given. The subnet $\left(x^{\beta^{\prime}}\right)_{\beta^{\prime} \in I^{\prime}}$ converges to $\bar{x}$. As such, there exists $\gamma^{\prime} \in I^{\prime}$ such that $x^{\gamma^{\prime}} \in B_{\varepsilon / 2}(\bar{x})$. In addition, $x^{\gamma^{\prime}} \in f^{\infty}(y)$, so there is $k \in \mathbb{N}$ and $x \in f^{k}(y)$ such that $x \in B_{\varepsilon / 2}\left(x^{\gamma^{\prime}}\right)$. Then, by the triangle inequality, it holds that $x \in B_{\varepsilon}(\bar{x})$. Together with $x \in f^{k}(y)$, this concludes the proof, that is, $\bar{x} \in f^{\infty}(y)$.

Q.E.D. 
Proof OF Lemma 3.2: Step 1: Let $\Gamma$ be a social environment and let $M$ be an MSS of $\Gamma$. We show in Step 1 that if $x \in M$ and $y \in f^{\infty}(x)$, then $y \in M$.

Let $x \in M$ and $y \in f^{\infty}(x)$ and suppose, toward a contradiction, that $y \notin M$. Given that $M$ is closed, there is $\varepsilon>0$ such that $B_{\varepsilon}(y) \cap M=\emptyset$. Also, by definition, there is $k \in \mathbb{N}$ and $z \in f^{k}(x)$ such that $z \in B_{\varepsilon}(y)$, that is, $z \notin M$. Since $z \in f^{k}(x)$, there is a sequence $z^{0}, z^{1}, \ldots, z^{k}$ of length $k$ such that

$$
z^{0}=x, \quad z^{1} \in f\left(z^{0}\right), \quad \ldots, \quad z^{k}=z \in f\left(z^{k-1}\right) .
$$

Let $k^{\prime} \in\{1, \ldots, k\}$ be such that $z^{k^{\prime}}$ is the first element in this sequence with the property that $z^{k^{\prime}} \notin M$. Given that $z^{0}=x \in M$ and $z^{k}=z \notin M$, such an element exists. It holds that $z^{k^{\prime}-1} \in M, z^{k^{\prime}} \in f\left(z^{k^{\prime}-1}\right)$, and $z^{k^{\prime}} \notin M$. This contradicts deterrence of external deviations for $M$.

Step 2: It remains to show that $M_{1} \cap M_{2} \neq \emptyset$.

Consider a state $x_{1} \in M_{1}$. If $x_{1} \in M_{2}$, then we are done. Otherwise, by asymptotic external stability of $M_{2}$, we know that there is $x_{2} \in M_{2}$ such that $x_{2} \in f^{\infty}\left(x_{1}\right)$. By Step 1 , we have that $x_{2} \in M_{1}$, so $x_{2} \in M_{1} \cap M_{2}$.

Q.E.D.

The following technical lemma is helpful in proving Theorem 3.4.

LEMMA A.1: If the dominance correspondence $f: X \rightarrow X$ is lower hemi-continuous, then the asymptotic dominance correspondence $f^{\infty}: X \rightarrow X$ is transitive.

ProOF: Let $x, y, z \in X$ be such that $y \in f^{\infty}(x)$ and $z \in f^{\infty}(y)$. We have to show that $z \in f^{\infty}(x)$, so we need to show that for every $\varepsilon>0$, there is $k^{\prime} \in \mathbb{N}$ and $z^{\prime} \in f^{k^{\prime}}(x)$ such that $z^{\prime} \in B_{\varepsilon}(z)$.

By assumption, $z \in f^{\infty}(y)$, so there is $k \in \mathbb{N}$ and $z_{1} \in f^{k}(y)$ such that $z_{1} \in B_{\varepsilon / 2}(z)$. In addition, as $y \in f^{\infty}(x)$, we know that for every $\ell \in \mathbb{N}$ there is $k_{\ell} \in \mathbb{N}$ and $y^{\ell} \in f^{k_{\ell}}(x)$ such that $y^{\ell} \in B_{1 / \ell}(y)$. This generates a sequence $\left(y^{\ell}\right)_{\ell \in \mathbb{N}}$ that converges to $y$, that is, $y^{\ell} \rightarrow y$.

Note that $f^{k}$ is lower hemi-continuous, since it is a composition of $k$ lower hemicontinuous correspondences. Given lower hemi-continuity of $f^{k}$ and the fact that $z_{1} \in$ $f^{k}(y)$, we know that there is a sequence $\left(z_{2}^{\ell}\right)_{\ell \in \mathbb{N}}$ such that $z_{2}^{\ell} \rightarrow z_{1}$ and $z_{2}^{\ell} \in f^{k}\left(y^{\ell}\right)$. Now, we have that $y^{\ell} \in f^{k_{\ell}}(x)$ and $z_{2}^{\ell} \in f^{k}\left(y^{\ell}\right)$, which gives $z_{2}^{\ell} \in f^{k+k_{\ell}}(x)$.

We take $\ell$ large enough such that $z_{2}^{\ell} \in B_{\varepsilon / 2}\left(z_{1}\right)$. Since $z_{1} \in B_{\varepsilon / 2}(z)$, the triangular inequality gives $z_{2}^{\ell} \in B_{\varepsilon}(z)$. This completes the proof.

Proof of Theorem 3.4: Suppose not, then, by Theorem 3.1 and Lemma 3.2, there exists a MSS $M_{1}$ and a MSS $M_{2}$ such that $M_{1} \neq M_{2}$ and their intersection $M_{3}=M_{1} \cap M_{2}$ is nonempty. Let us show that $M_{3}$ is a QMSS, contradicting the minimality of $M_{1}$ and $M_{2}$, and establishing the uniqueness of the MSS. First of all, notice that $M_{3}$, being the intersection of two closed sets, is also closed.

For deterrence of external deviations, let $x \in M_{3}$ and $y \in f(x)$. Then given that $x \in M_{1}$ and $M_{1}$ satisfies deterrence of external deviations, it must be that $y \in M_{1}$. Also given that $x \in M_{2}$ and $M_{2}$ satisfies deterrence of external deviations, it must be that $y \in M_{2}$. This implies that $y \in M_{1} \cap M_{2}=M_{3}$ as was to be shown.

For asymptotic external stability, take any $y \notin M_{3}$. There are three cases to consider.

Case 1: $y \in M_{1} \backslash M_{3}$ : Then, by asymptotic external stability of $M_{2}$, there is $x \in M_{2}$ such that $x \in f^{\infty}(y)$. By Step 1 in the proof of Lemma 3.2, we have that $x \in M_{1}$. This means that $x \in M_{1} \cap M_{2}=M_{3}$, which is what we needed to show. 
Case 2: $y \in M_{2} \backslash M_{3}$ : The proof is symmetric to Case 1 with $M_{1}$ and $M_{2}$ interchanged.

Case 3: $y \in X \backslash\left(M_{1} \cup M_{2}\right)$ : We know, by asymptotic external stability of $M_{1}$, that there is $x \in M_{1}$ such that $x \in f^{\infty}(y)$. If $x \in M_{3}$, we are done. If not, we know from Case 1 above that there is $z \in M_{3}$ such that $z \in f^{\infty}(x)$. It follows from $x \in f^{\infty}(y)$ and $z \in f^{\infty}(x)$ that $z \in f^{\infty}(y)$ by Lemma A.1.

Q.E.D.

Proof of Theorem 3.7: Let $x, y \in X$ and sequences $\left(x^{k}\right)_{k \in \mathbb{N}}$ and $\left(y^{k}\right)_{k \in \mathbb{N}}$ in $X$ such that $x^{k} \rightarrow x$ and $y^{k} \rightarrow y$ be given. Let us first show that if individual $i \in N$ strictly prefers $y$ to $x$, so $y \succ_{i} x$, then there is $\ell \in \mathbb{N}$ such that for all $k \geq \ell, y^{k} \succ_{i} x^{k}$. Suppose not, then for every $\ell \in \mathbb{N}$ we can find $k_{\ell} \geq \ell$ such that $x^{k_{\ell}} \succeq_{i} y^{k_{\ell}}$. This creates sequences $\left(x^{k_{\ell}}\right)_{\ell \in \mathbb{N}}$, $\left(y^{k_{\ell}}\right)_{\ell \in \mathbb{N}}$ in $X$ with $x^{k_{\ell}} \rightarrow x$ and $y^{k_{\ell}} \rightarrow y$ such that, for every $\ell \in \mathbb{N}, x^{k_{\ell}} \succeq_{i} y^{k_{\ell}}$. By continuity of $\succeq_{i}$, it holds that $x \succeq_{i} y$, a contradiction.

Let $\left(x^{k}\right)_{k \in \mathbb{N}}$ be a sequence in $X$ such that $x^{k} \rightarrow x$ and consider some $y \in f(x)$. Then either $y=x$ or $y \neq x$ and there is a coalition $S \in E(x, y)$ such that, for every $i \in S, y \succ_{i} x$.

If $y=x$, take the sequence $\left(y^{k}\right)_{k \in \mathbb{N}}$ in $X$ defined by $y^{k}=x^{k}$. We immediately have that, for every $k \in \mathbb{N}, y^{k} \in f\left(x^{k}\right)$, and $y^{k} \rightarrow y$.

We now consider the case where $y \neq x$ and there is a coalition $S \in E(x, y)$ such that, for every $i \in S, y \succ_{i} x$. By lower hemi-continuity of the correspondence $G_{S}$, we know that there is a sequence $\left(y^{k}\right)_{k \in \mathbb{N}}$ such that $y^{k} \in G_{S}\left(x^{k}\right)$ and $y^{k} \rightarrow y$. By the first paragraph of the proof, we know that for every $i \in S$ there is $\ell_{i} \in \mathbb{N}$ such that, for every $k \geq \ell_{i}, y^{k} \succ_{i} x^{k}$. Let $\ell=\max _{i \in S} \ell_{i}$. Then, for every $k \geq \ell$, for every $i \in S, y^{k} \succ_{i} x^{k}$, and $S \in E\left(\bar{x}^{k}, y^{k}\right)$, which shows that $y^{k} \in f\left(x^{k}\right)$. The sequence $\left(z^{k}\right)_{k \in \mathbb{N}}$ defined by $z^{k}=x^{k}$ if $k<\ell$ and $z^{k}=y^{k}$ if $k \geq \ell$ therefore has all the desired properties: for every $k \in \mathbb{N}, z^{k} \in f\left(x^{k}\right)$, and $z^{k} \rightarrow y$.

As $f$ is lower hemi-continuous, Theorem 3.4 implies that the social environment has a unique MSS.

Q.E.D.

Proof OF THEOREM 3.9: Toward a contradiction, suppose there is a closed cycle $C$ which is not a subset of $M$. Let $x \in C$ and $x \notin M$. By asymptotic external stability there is $y \in M$ such that $y \in f^{\infty}(x)$. By definition of a closed cycle, it follows that $y \in C$. As $x \in C$, again by definition of a closed cycle, we also have that $x \in f^{\infty}(y)$. By Step 1 in the proof of Lemma 3.2, it follows that $x \in M$, a contradiction. Since the choice of $C$ was arbitrary, we have shown that $\mathrm{CC} \subseteq M$.

We show next that if $X$ is finite, then $\mathrm{CC}=M$. Since $\mathrm{CC} \subseteq M$, we only need to show that CC is a QMSS. The set CC satisfies deterrence of external deviations, since for all $x \in \mathrm{CC}, f(x) \subseteq f^{\infty}(x) \subseteq \mathrm{CC}$. It remains to verify asymptotic external stability of CC, that is, for every state $x \notin \mathrm{CC}, f^{\infty}(x) \cap \mathrm{CC} \neq \emptyset$.

Let $x \notin \mathrm{CC}$ and define $Y=f^{\infty}(x)$. Note that $Y$ is nonempty since $x \in f(x)$. It also holds that $Y$ is finite and $f^{\infty}(y) \subseteq Y$ for every $y \in Y$. Let us represent the set $Y$ and the dominance correspondence $f$ on $Y$ by a finite directed graph $D$, that is, (i) $Y$ is the set of vertices of $D$ and (ii) $D$ has an arc from $y$ to $z$ if and only if $z \in f(y)$. By contracting each strongly connected component of $D$ to a single vertex, we obtain a directed acyclic graph, which is called the condensation of $D$. As the condensation is finite and acyclic, it has a maximal element, say $c$. Observe that $c$ represents a closed cycle $C$, so $Y \cap \mathrm{CC} \neq \emptyset$.

Q.E.D.

ProOf OF THEOREM 3.13: Assume that $\Gamma$ satisfies the weak improvement property. Let $M$ be an MSS of $\Gamma$. By Corollary 3.11, we have $\mathrm{CO} \subseteq M$. We will show that $\mathrm{CO}$ is a QMSS. By minimality, it then follows that $\mathrm{CO}=M$.

In order to see that $\mathrm{CO}$ is closed, let $\left(x^{k}\right)_{k \in \mathbb{N}}$ be a sequence in $\mathrm{CO}$, so for all $k \in \mathbb{N}$ it holds that $f\left(x^{k}\right)=\left\{x^{k}\right\}$. Now assume that $x^{k} \rightarrow x$ and $x \notin \mathrm{CO}$. This means that there is 
$y \neq x$ such that $y \in f(x)$. By lower hemi-continuity of $f$, there is a sequence $\left(y^{k}\right)_{k \in \mathbb{N}}$ such that $y^{k} \in f\left(x^{k}\right)$ and $y^{k} \rightarrow y$. As for all $k \in \mathbb{N}, x^{k} \in \mathrm{CO}$, we have that $y^{k}=x^{k}$, which means that $y^{k} \rightarrow x$, so $y=x$, a contradiction. Deterrence of external deviations is immediate for the core as it is the union of the sinks. If the social environment satisfies the weak improvement property, we have that for all $x \notin \mathrm{CO}, f^{\infty}(x) \cap \mathrm{CO} \neq \emptyset$, thus the core satisfies asymptotic external stability.

For the reverse, let $M$ be a MSS equal to CO. Now, if $x \in M$, it is a sink, so $f^{\infty}(x)=$ $\{x\} \subseteq \mathrm{CO}$. If $x \notin \mathrm{CO}$, we have by asymptotic external stability of $M$ that $f^{\infty}(x) \cap M \neq \emptyset$, so $f^{\infty}(x)$ contains a sink, that is, $\Gamma$ satisfies the weak improvement property.

Q.E.D.

Proof OF THEOREM 3.15: Suppose that $M$ is a MSS such that $M \cap V=\emptyset$. Recall that $M$ is nonempty. Let $x \in M$ and $x \notin V$. Since $V$ satisfies external stability, there is $y \in f(x)$ such that $y \in V$. Since $M$ satisfies deterrence of external deviations, we have that $y \in M$, so $M \cap V \neq \emptyset$, a contradiction.

Q.E.D.

PROOF OF THEOREM 3.16: By Theorem 3.9, the MSS is unique and equal to the union of all closed cycles CC. Since $Q$ is consistent with $f$, a state is recurrent if and only if it belongs to a closed cycle.

Q.E.D.

\section{PROOF OF THEOREM 4.4:}

Step 1: $\Gamma$ has a unique MSS.

We show lower hemi-continuity of $E$ and continuity of the preferences. The result then follows from Theorem 3.7.

To show lower hemi-continuity of $E$, let some $S \in \mathcal{N}$, a sequence $\left(x^{k}\right)_{k \in \mathbb{N}}$ in $X$ such that $x^{k} \rightarrow x$, and some $y \in G_{S}(x)$ be given. We show that there is a sequence $\left(y^{k}\right)_{k \in \mathbb{N}}$ such that $y^{k} \in G_{S}\left(x^{k}\right)$ and $y^{k} \rightarrow y$. If $y=x$, then the choice $y^{k}=x^{k}$ would do, so consider the case $y \neq x$.

First of all, there is $k^{\prime} \in \mathbb{N}$ such that for all $k \geq k^{\prime}, \pi\left(x^{k}\right)=\pi(x)$, so in particular $\mathcal{U}\left(x^{k}, S\right)=\mathcal{U}(x, S)$. For every $k<k^{\prime}$, we define $y^{k}=x^{k}$. For every $k \geq k^{\prime}$, we define $y^{k} \in X$ by $\pi\left(y^{k}\right)=\pi(y)$ and

$$
u_{i}\left(y^{k}\right)= \begin{cases}u_{i}(y), & i \in N \backslash U(x, S), \\ u_{i}\left(x^{k}\right), & i \in U(x, S) .\end{cases}
$$

This completely specifies the state $y^{k}$. Consider some $k \geq k^{\prime}$. Since $y \neq x$ and $y \in G_{S}(x)$, it holds that $S \in \pi(y)$ and, for every $i \in N \backslash(S \cup U(x, S))$, we have that $i$ is a residual player. The properties of the $\gamma$-model imply that $\{i\} \in \pi(y)$. The same properties hold for $\pi\left(y^{k}\right)$. For every $i \in S$, it holds that $u_{i}\left(y^{k}\right)=u_{i}(y)$, so $u_{i}\left(y^{k}\right) \geq v(\{i\})$ and $\sum_{i \in S} u_{i}\left(y^{k}\right)=v(S)$. For every $i \in N \backslash(S \cup U(x, S))$, we have that $u_{i}\left(y^{k}\right)=v(\{i\})=u_{i}(y)$. For every $i \in U(x, S)$ it holds that $u_{i}(y)=u_{i}(x)$ and $u_{i}\left(y^{k}\right)=u_{i}\left(x^{k}\right)$. By coalitional sovereignty, we have that $y^{k} \in G_{S}\left(x^{k}\right)$. Using that $x^{k} \rightarrow x$, it follows easily that $y^{k} \rightarrow y$.

Let some $i \in N$ be given. To show continuity of $\succeq_{i}$, let $\left(x^{k}\right)_{k \in \mathbb{N}}$ and $\left(y^{k}\right)_{k \in \mathbb{N}}$ be sequences in $X$ such that $x^{k} \rightarrow x$ and $y^{k} \rightarrow y$. Then, by continuity of the projection, we have that $u_{i}\left(x^{k}\right) \rightarrow u_{i}(x)$ and $u_{i}\left(y^{k}\right) \rightarrow u_{i}(y)$. So if $u_{i}\left(x^{k}\right) \geq u_{i}\left(y^{k}\right)$ for all $k \in \mathbb{N}$, we obtain $u_{i}(x) \geq$ $u_{i}(y)$, which shows that $x \succeq_{i} y$.

Step 2: The core of $\Gamma$ is equal to $Y$.

Let $y \in \mathrm{CO}$ and suppose $y \notin Y$. Then there is a coalition $S \in \mathcal{N}$ such that $\sum_{i \in S} u_{i}(y)<$ $v(S)$. Since $y \in X$, it holds for all $i \in S$ that $u_{i}(y) \geq v(\{i\})$. Now, let $u_{S}$ be a vector of payoffs for the members in $S$ such that $\sum_{i \in S} u_{i}=v(S)$ and for all $i \in S, u_{i}>u_{i}(y)$. Then, 
by full support, there exists a state $y^{\prime} \in X$ such that $S \in E\left(y, y^{\prime}\right)$ and $u_{S}=u_{S}\left(y^{\prime}\right)$. We conclude that $y^{\prime} \in f(y)$. This contradicts the fact that $y \in \mathrm{CO}$.

For the reverse, let $y \in Y$ and suppose there is $z \in f(y)$ such that $z \neq y$, that is, $y \notin$ $\mathrm{CO}$. Then there is $S \in E(y, z)$ such that for all $i \in S$ it holds that $u_{i}(z)>u_{i}(y)$. Also, $v(S)=\sum_{i \in S} u_{i}(z)>\sum_{i \in S} u_{i}(y) \geq v(S)$, where the first equality follows from the definition of the state space and the last inequality from the definition of $Y$. We have obtained a contradiction.

Step 3: If $Y \neq \emptyset$, then the MSS of $\Gamma$ is equal to $Y$.

Step 2 shows that $Y$ is equal to the core of $\Gamma$. If we can show that $\Gamma$ satisfies the weak improvement property whenever $Y \neq \emptyset$, then we can use Theorem 3.13 to establish our result. Since the proof is trivial when the number of individuals $n$ is equal to 1 , we assume $n \geq 2$ throughout.

Assume that $Y \neq \emptyset$. We need to show that for all $x^{0} \in X, f^{\infty}\left(x^{0}\right) \cap Y \neq \emptyset$. If $x^{0}$ in $Y$, then nothing needs to be shown, so assume that $x^{0} \in X \backslash Y$. We need to show that for every $\varepsilon>0$ there is a number $k^{\prime} \in \mathbb{N}$, a state $x^{k^{\prime}} \in f^{k^{\prime}}\left(x^{0}\right)$, and a state $y \in Y$ such that $d\left(x^{k^{\prime}}, y\right)<\varepsilon$.

Let some $\varepsilon>0$ be given. Béal, Rémila, and Solal (2013) show that there exists a sequence of states $\left(x^{0}, \ldots, x^{k^{\prime}}\right)$ such that $x^{k^{\prime}} \in Y, k^{\prime}$ is less than or equal to $\left(n^{2}+4 n\right) / 4$, and, for every $k \in\left\{1, \ldots, k^{\prime}\right\}$ :

1. there is $S^{k} \in \mathcal{N}$ such that $S^{k} \in E\left(x^{k-1}, x^{k}\right)$,

2. $u_{S^{k}}\left(x^{k-1}\right)<u_{S^{k}}\left(x^{k}\right)$.

Notice that the inequality in 2. only means that at least one of the players in $S^{k}$ gets a strictly higher payoff, not necessarily all of them. Let $P^{k}$ be the set of partners of the players in $S^{k}$ at state $x^{k-1}$, more formally defined as

$$
P^{k}=\bigcup_{\left\{S \in \pi\left(x^{k-1}\right) \mid S \cap S^{k} \neq \emptyset\right\}} S,
$$

so $P^{k}$ is equal to the moving coalition $S^{k}$ together with the residual players. Since $S^{k} \in$ $E\left(x^{k-1}, x^{k}\right)$, it follows that

$$
\begin{aligned}
& u_{i}\left(x^{k}\right)=v(\{i\}), \quad i \in P^{k} \backslash S^{k}, \\
& u_{i}\left(x^{k}\right)=u_{i}\left(x^{k-1}\right), \quad i \in N \backslash P^{k} .
\end{aligned}
$$

We define $W^{k} \subset S^{k}$ to be the, possibly empty, proper subset of $S^{k}$ consisting of players that only weakly improve when moving from state $x^{k-1}$ to state $x^{k}$, so for every $i \in W^{k}$ it holds that $u_{i}\left(x^{k-1}\right)=u_{i}\left(x^{k}\right)$. We define

$$
\delta=\min _{k \in\left\{1, \ldots, k^{\prime}\right\}} \min _{i \in S^{k} \backslash W^{k}} u_{i}\left(x^{k}\right)-u_{i}\left(x^{k-1}\right) \quad \text { and } \quad \varepsilon^{\prime}=\min \{\delta, \varepsilon\},
$$

so $\delta$ is the smallest improvement of any of the strictly improving players involved in any move along the sequence. It holds that $\delta>0$ and, therefore, that $\varepsilon^{\prime}>0$. For $k \in\left\{0, \ldots, k^{\prime}\right\}$, define $\nu_{k}=n^{2 k} /\left(n^{2 k^{\prime}+1}\right)$. We define $e\left(W^{k}\right)=0$ if $W^{k}=\emptyset$ and $e\left(W^{k}\right)=1$ otherwise. We use the sequence $\left(x^{0}, x^{1}, \ldots, x^{k^{\prime}}\right)$ of states as constructed by Béal, Rémila, and Solal (2013) to define a new sequence $\left(\widetilde{x}^{0}, \widetilde{x}^{1}, \ldots, \widetilde{x}^{k^{\prime}}\right)$ of states by setting $\widetilde{x}^{0}=x^{0}$ and, for every $k \in\left\{1, \ldots, k^{\prime}\right\}$,

$$
\pi\left(\widetilde{x}^{k}\right)=\pi\left(x^{k}\right)
$$




$$
\begin{aligned}
& u_{i}\left(\tilde{x}^{k}\right)=u_{i}\left(x^{k}\right)+\varepsilon^{\prime} \nu_{k} \frac{\left|S^{k} \backslash W^{k}\right|}{\left|W^{k}\right|}, \quad i \in W^{k}, \\
& u_{i}\left(\tilde{x}^{k}\right)=u_{i}\left(x^{k}\right)-\varepsilon^{\prime} \nu_{k} e\left(W^{k}\right), \quad i \in S^{k} \backslash W^{k}, \\
& u_{i}\left(\widetilde{x}^{k}\right)=u_{i}\left(x^{k}\right)=v(\{i\}), \quad i \in P^{k} \backslash S^{k}, \\
& u_{i}\left(\tilde{x}^{k}\right)=u_{i}\left(\tilde{x}^{k-1}\right), \quad i \in N \backslash P^{k} .
\end{aligned}
$$

Notice that the first line does not entail a division by zero, since if $i \in W^{k}$, then $W^{k} \neq \emptyset$.

Compared to the sequence $\left(x^{0}, x^{1}, \ldots, x^{k^{\prime}}\right)$, the sequence $\left(\tilde{x}^{0}, \tilde{x}^{1}, \ldots, \widetilde{x}^{k^{\prime}}\right)$ is such that each strictly improving player in $S^{k} \backslash W^{k}$ donates an amount $\varepsilon^{\prime} \nu_{k} /\left|W^{k}\right|$ to each of the players in $W^{k}$ whenever the latter set is nonempty. It is also important to observe that the fraction $\nu_{k}$ is an $n^{2}$ multiple of $\nu_{k-1}$ and that $\nu_{k^{\prime}}=1 / n$.

We show first by induction that, for every $k \in\left\{0, \ldots, k^{\prime}\right\}, \widetilde{x}^{k} \in X$. Obviously, it holds that $\tilde{x}^{0}=x^{0} \in X$. Assume that, for some $k \in\left\{1, \ldots, k^{\prime}\right\}, \widetilde{x}^{k-1} \in X$. We show that $\tilde{x}^{k} \in X$. It holds that

$$
\begin{aligned}
& u_{i}\left(\tilde{x}^{k}\right)>u_{i}\left(x^{k}\right) \geq v(\{i\}), \quad i \in W^{k}, \\
& u_{i}\left(\tilde{x}^{k}\right) \geq u_{i}\left(x^{k-1}\right)+\delta-\varepsilon^{\prime} \nu_{k}>u_{i}\left(x^{k-1}\right)+\delta-\varepsilon^{\prime} \geq u_{i}\left(x^{k-1}\right) \geq v(\{i\}), \quad i \in S^{k} \backslash W^{k}, \\
& u_{i}\left(\tilde{x}^{k}\right)=v(\{i\}), \quad i \in P^{k} \backslash S^{k}, \\
& u_{i}\left(\tilde{x}^{k}\right)=u_{i}\left(\tilde{x}^{k-1}\right) \geq v(\{i\}), \quad i \in N \backslash P^{k},
\end{aligned}
$$

where the very last inequality follows from the induction hypothesis. Moreover, for every $S \in \pi\left(x^{k}\right)$, it holds that either $S=S^{k}$ and $W^{k}=\emptyset$, so

$$
\sum_{i \in S} u_{i}\left(\widetilde{x}^{k}\right)=\sum_{i \in S^{k}} u_{i}\left(x^{k}\right)=v(S)
$$

or $S=S^{k}$ and $W^{k} \neq \emptyset$, so

$$
\sum_{i \in S} u_{i}\left(\tilde{x}^{k}\right)=\sum_{i \in W^{k}}\left(u_{i}\left(x^{k}\right)+\varepsilon^{\prime} \nu_{k} \frac{\left|S^{k} \backslash W^{k}\right|}{\left|W^{k}\right|}\right)+\sum_{i \in S^{k} \backslash W^{k}}\left(u_{i}\left(x^{k}\right)-\varepsilon^{\prime} \nu_{k}\right)=\sum_{i \in S^{k}} u_{i}\left(x^{k}\right)=v(S),
$$

or $S=\left\{i^{\prime}\right\}$ with $i^{\prime} \in P^{k} \backslash S^{k}$ and

$$
\sum_{i \in S} u_{i}\left(\tilde{x}^{k}\right)=u_{i^{\prime}}\left(\tilde{x}^{k}\right)=u_{i^{\prime}}\left(x^{k}\right)=v\left(\left\{i^{\prime}\right\}\right)=v(S)
$$

or $S \subseteq N \backslash P^{k}$, so $S \in \pi\left(\tilde{x}^{k-1}\right)$, and

$$
\sum_{i \in S} u_{i}\left(\tilde{x}^{k}\right)=\sum_{i \in S} u_{i}\left(\tilde{x}^{k-1}\right)=v(S)
$$

where the last equality makes use of the induction hypothesis. We have now completed the proof of the fact that for every $k \in\left\{0, \ldots, k^{\prime}\right\}, \widetilde{x}^{k} \in X$.

We show next by induction that, for every $k \in\left\{0, \ldots, k^{\prime}\right\}$, and for every $i \in N$,

$$
\left|u_{i}\left(\tilde{x}^{k}\right)-u_{i}\left(x^{k}\right)\right| \leq \varepsilon^{\prime} \nu_{k}(n-1) .
$$


Obviously, for every $i \in N$, it holds that $\left|u_{i}\left(\tilde{x}^{0}\right)-u_{i}\left(x^{0}\right)\right|=0 \leq \varepsilon^{\prime} \nu_{0}(n-1)$. Assume that, for some $k \in\left\{1, \ldots, k^{\prime}\right\}$, for every $i \in N,\left|u_{i}\left(\widetilde{x}^{k-1}\right)-u_{i}\left(x^{k-1}\right)\right| \leq \varepsilon^{\prime} \nu_{k-1}(n-1)$. We show that, for every $i \in N,\left|u_{i}\left(\widetilde{x}^{k}\right)-u_{i}\left(x^{k}\right)\right| \leq \varepsilon^{\prime} \nu_{k}(n-1)$. If $i \in W^{k}$, then $W^{k} \neq \emptyset$, and the statement follows from the observation that

$$
0 \leq u_{i}\left(\widetilde{x}^{k}\right)-u_{i}\left(x^{k}\right)=\varepsilon^{\prime} \nu_{k} \frac{\left|S^{k} \backslash W^{k}\right|}{\left|W^{k}\right|} \leq \varepsilon^{\prime} \nu_{k}(n-1) .
$$

If $i \in S^{k} \backslash W^{k}$, then we have that

$$
0 \geq u_{i}\left(\widetilde{x}^{k}\right)-u_{i}\left(x^{k}\right) \geq-\varepsilon^{\prime} \nu_{k} \geq-\varepsilon^{\prime} \nu_{k}(n-1) .
$$

If $i \in P^{k} \backslash S^{k}$, then we have $\left|u_{i}\left(\tilde{x}^{k}\right)-u_{i}\left(x^{k}\right)\right|=0$. If $i \in N \backslash P^{k}$, then it holds that

$$
\left|u_{i}\left(\widetilde{x}^{k}\right)-u_{i}\left(x^{k}\right)\right|=\left|u_{i}\left(\widetilde{x}^{k-1}\right)-u_{i}\left(x^{k-1}\right)\right| \leq \varepsilon^{\prime} \nu_{k-1}(n-1)<\varepsilon^{\prime} \nu_{k}(n-1),
$$

where the first inequality makes use of the induction hypothesis and the last inequality of the fact that $\nu_{k-1}<\nu_{k}$.

Let some $k \in\left\{1, \ldots, k^{\prime}\right\}$ and some $i \in S^{k}$ be given. We show that $u_{i}\left(\widetilde{x}^{k}\right)>u_{i}\left(\widetilde{x}^{k-1}\right)$. If $i \in W^{k}$, then it holds that

$$
\begin{aligned}
u_{i}\left(\widetilde{x}^{k}\right) & =u_{i}\left(x^{k}\right)+\varepsilon^{\prime} \nu_{k} \frac{\left|S^{k} \backslash W^{k}\right|}{\left|W^{k}\right|}=u_{i}\left(x^{k-1}\right)+\varepsilon^{\prime} \nu_{k} \frac{\left|S^{k} \backslash W^{k}\right|}{\left|W^{k}\right|} \\
& \geq u_{i}\left(\tilde{x}^{k-1}\right)-\varepsilon^{\prime} \nu_{k-1}(n-1)+\varepsilon^{\prime} \nu_{k} \frac{1}{n-1}>u_{i}\left(\widetilde{x}^{k-1}\right),
\end{aligned}
$$

where the strict inequality uses that $\nu_{k}=n^{2} \nu_{k-1}$. If $i \in S^{k} \backslash W^{k}$, then it holds that

$$
\begin{aligned}
u_{i}\left(\widetilde{x}^{k}\right) & \geq u_{i}\left(x^{k}\right)-\varepsilon^{\prime} \nu_{k} \geq u_{i}\left(x^{k-1}\right)+\delta-\varepsilon^{\prime} \nu_{k} \\
& \geq u_{i}\left(\widetilde{x}^{k-1}\right)-\varepsilon^{\prime} \nu_{k-1}(n-1)+\delta-\varepsilon^{\prime} n^{2} \nu_{k-1}>u_{i}\left(\widetilde{x}^{k-1}\right),
\end{aligned}
$$

where the strict inequality uses the facts that $\delta \geq \varepsilon^{\prime}$ and

$$
\left(n^{2}+(n-1)\right) \nu_{k-1}<2 n^{2} \nu_{k-1} \leq 2 \nu_{k} \leq 1 .
$$

Combining the statements proven so far, it follows that $\widetilde{x}^{k^{\prime}} \in f^{k^{\prime}}\left(x^{0}\right)$. We complete the proof of the weak improvement property by noting that $x^{k^{\prime}} \in Y$ by the result of Béal, Rémila, and Solal (2013) and by observing that $d\left(\widetilde{x}^{k^{\prime}}, x^{k^{\prime}}\right)<\varepsilon$ since $\pi\left(\widetilde{x}^{k^{\prime}}\right)=\pi\left(x^{k^{\prime}}\right)$ and, for every $i \in N$,

$$
\left|u_{i}\left(\widetilde{x}^{k^{\prime}}\right)-u_{i}\left(x^{k^{\prime}}\right)\right| \leq \varepsilon^{\prime} \nu_{k^{\prime}}(n-1)<\varepsilon^{\prime} \leq \varepsilon .
$$

Proof OF THEOREM 4.5: We show first that $F(X)$ satisfies deterrence of external deviations. Let $x \in F(X)$ and $y \in f(x)$ be given and let $S \in E(x, y)$ be such that, for every $i \in S, u_{i}(y)>u_{i}(x)$. It follows that $S \in \mathcal{W}$. Since $\sum_{i \in N} u_{i}(x)=\sum_{i \in N} u_{i}(y)=1$, we have that $S \neq N$. Since $N \backslash S \notin \mathcal{W}, S$ has a nonempty intersection with the unique coalition in $\pi(x) \cap \mathcal{W}$. It follows that $\pi(y)=\{S\} \cup\{\{i\} \mid i \in N \backslash S\}$, so it holds that $y \in F(X)$.

We show next that $F(X)$ satisfies asymptotic external stability. Let some $x \in X \backslash F(X)$ be given. If $\pi(x) \cap \mathcal{W} \neq \emptyset$, then let $j \in N$ be such that $u_{j}(x)>0$. If $\pi(x) \cap \mathcal{W}=\emptyset$, then 
take any $j \in N$. Since the core of $v$ is empty, it holds that $N \backslash\{j\} \in \mathcal{W}$, as otherwise $j$ would be a veto player. Let $y \in F(X)$ be such that $\pi(y)=\{N \backslash\{j\},\{j\}\}$ and $u_{N \backslash\langle j\}}(y) \gg u_{N \backslash\{j\}}(x)$. Since $N \backslash\{j\} \in E(x, y)$ it holds that $y \in f(x)$.

We show next that for every $x \in F(X)$ it holds that $f^{\infty}(x)=F(X)$. Let some $x, y \in$ $F(X)$ be given and denote the winning coalition in $\pi(y)$ by $W$. We have to show that for every $\varepsilon>0$ there exists $k^{\prime} \in \mathbb{N}$ and $z \in f^{k^{\prime}}(x)$ such that $z \in B_{\varepsilon}(y)$.

Let some $\varepsilon \in(0,1 / n)$ and $S \in \pi(y) \backslash\{W\}$ be given. We show first that there is $k \in \mathbb{N}$ and $x^{k} \in f^{k}(x)$ such that for every $i \in N \backslash S, u_{i}\left(x^{k}\right)<\varepsilon / n$ and each element of $\pi\left(x^{k}\right)$ is either a winning coalition or a singleton. If $x$ itself satisfies these properties, then we are done. Otherwise, there is $j \in N \backslash S$ such that $u_{j}(x) \geq \varepsilon / n$. Since $j$ is not a veto player, it holds that $N \backslash\{j\} \in \mathcal{W}$. Let $x^{1} \in X$ be such that $\pi\left(x^{1}\right)=\{N \backslash\{j\},\{j\}\}, u_{N \backslash\{j\}}\left(x^{1}\right) \gg u_{N \backslash\{j\}}(x)$, and, for every $i \in N \backslash\{j\}$ such that $u_{i}(x)<\varepsilon / n, u_{i}\left(x^{1}\right)<\varepsilon / n$. Since $u_{j}(x) \geq \varepsilon / n$, such an element $x^{1}$ exists. It holds that $x^{1} \in f(x)$ and $u_{j}\left(x^{1}\right)=0$. If there is $j^{1} \in N \backslash S$ such that $u_{j^{1}}\left(x^{1}\right) \geq \varepsilon / n$, then we repeat this argument using $j^{1}$. Since the set $N \backslash S$ is finite, we reach a state $x^{k}$ with the desired properties in a finite number of steps.

We define the possibly empty set $W^{0}=\left\{i \in W \mid u_{i}(y) \leq \varepsilon / n\right\}$. Let $w \in W$ be a player such that $u_{w}(y) \geq 1 / n$. Let $z \in X$ be such that $\pi(z)=\pi(y)=\{W\} \cup\{\{i\} \mid i \in N \backslash W\}$ and

$$
u_{j}(z)= \begin{cases}\frac{\varepsilon}{n}, & j \in W^{0}, \\ u_{j}(y), & j \in W \backslash\left(W^{0} \cup\{w\}\right), \\ u_{w}(y)-\sum_{i \in W^{0}}\left(\frac{\varepsilon}{n}-u_{i}(y)\right), & j=w .\end{cases}
$$

For every $j \in W^{0}$ it holds that $u_{j}(z)=\varepsilon / n>u_{j}\left(x^{k}\right)$, for every $j \in W \backslash\left(W^{0} \cup\{w\}\right)$ it holds that $u_{j}(z)=u_{j}(y)>\varepsilon / n>u_{j}\left(x^{k}\right)$, and

$$
u_{w}(z)=u_{w}(y)-\sum_{i \in W^{0}}\left(\frac{\varepsilon}{n}-u_{i}(y)\right) \geq \frac{1}{n}-\frac{n-2}{n} \frac{\varepsilon}{n}>\frac{\varepsilon}{n}>u_{w}\left(x^{k}\right),
$$

so $u_{W}(z) \gg u_{W}\left(x^{k}\right)$. Since every element of $\pi\left(x^{k}\right)$ is either a winning coalition or a singleton, it holds that $W \in E\left(x^{k}, z\right)$. We have shown that $z \in f\left(x^{k}\right)$ and therefore $z \in f^{k+1}(x)$. We have that $\pi(y)=\pi(z)$, for every $j \in W^{0}$ it holds that $\left|u_{j}(y)-u_{j}(z)\right| \leq \varepsilon / n$, for every $j \in W \backslash\left(W^{0} \cup\{w\}\right)$ it holds that $\left|u_{j}(y)-u_{j}(z)\right|=0$, and $\left|u_{w}(y)-u_{w}(z)\right| \leq(n-2) \varepsilon / n$, therefore, $z \in B_{\varepsilon}(y)$, so $z$ has all the desired properties.

It follows by Theorem 3.9 that $F(X)$ is a subset of the MSS and since $F(X)$ satisfies deterrence of external deviations and asymptotic external stability, it must be equal to the MSS.

Q.E.D.

ProOf OF THEOREM 4.10: It is easy to see that the effectivity correspondence $E$ is lower hemi-continuous. Moreover, continuity of $\left(\succeq_{i}\right)_{i \in N}$ is identical to continuity of the utility functions $\left(u_{i}\right)_{i \in N}$. As such, it suffices to show that $\Gamma$ satisfies the weak improvement property.

Consider a function $b: \Sigma \rightarrow \Sigma$ such that, for every $s \in \Sigma$,

$$
b(s) \in \underset{\left\{\tau \in \Sigma|| i \in \in N, \tau_{-i}=s_{-i}\right\}}{\arg \max } P(\tau),
$$

and $b(s)=s$ if $P(b(s))=P(s)$. For each strategy profile $s \in \Sigma$, there is $j \in N$ and $\tau_{j}^{*} \in \Sigma_{j}$ such that $b(s)=\left(\tau_{j}^{*}, s_{-j}\right)$ and $b(s)$ maximizes the value of $P(\tau)$ over all strategies $\tau$ such 
that there is $i \in N$ with $\tau_{-i}=s_{-i}$. If the maximal value of $P(\tau)$ is equal to $P(s)$, then $b(s)$ is taken equal to $s$. As $P$ is continuous and, for every $i \in N, \Sigma_{i}$ is compact, the maximization problem has a solution.

Observe that $s \in \Sigma$ is a pure strategy Nash equilibrium of $G$ if and only if

$$
P(s) \geq P\left(\tau_{i}, s_{-i}\right), \quad i \in N, \tau_{i} \in \Sigma_{i} .
$$

It follows that $s$ is a pure strategy Nash equilibrium of $G$ if and only if $b(s)=s$. Let NE be the set of all pure strategy Nash equilibria of $G$. The set NE is nonempty as it contains all the strategy profiles that maximize $P$ over $\Sigma$, a nonempty set since $P$ is continuous and $\Sigma$ is compact.

Let us show that $\Gamma$ satisfies the weak improvement property, that is, for all states $s \in \Sigma$, $f^{\infty}(s) \cap \mathrm{NE} \neq \emptyset$.

Let $s^{1} \in \Sigma \backslash$ NE be given. Consider the sequence of states $\left(s^{k}\right)_{k \in \mathbb{N}}$ in $\Sigma$ defined by $s^{2}=$ $b\left(s^{1}\right), s^{3}=b\left(s^{2}\right), \ldots$ For every $k \in \mathbb{N}$, it holds that $s^{k+1} \in f\left(s^{k}\right)$, so any accumulation point of the sequence $\left(s^{k}\right)_{k \in \mathbb{N}}$ belongs to $f^{\infty}\left(s^{1}\right)$.

Observe that, by definition, $P\left(s^{1}\right) \leq P\left(s^{2}\right) \leq P\left(s^{3}\right) \leq \cdots$, so the sequence $\left(P\left(s^{k}\right)\right)_{k \in \mathbb{N}}$ is nondecreasing. Further, as $\left(s^{k}\right)_{k \in \mathbb{N}}$ takes values in the compact set $\Sigma$, it has a convergent subsequence. Let us denote such a subsequence by $\left(x^{\ell}\right)_{\ell \in \mathbb{N}}$ and let $x^{\ell} \rightarrow x$. It holds that $x \in f^{\infty}\left(s^{1}\right)$. By continuity of $P$, it holds that $P\left(x^{\ell}\right) \rightarrow P(x)$. It also holds that, for every $k \in \mathbb{N}, P\left(s^{k}\right) \leq P(x)$.

Since $x \in f^{\infty}\left(s_{1}\right)$, we complete the proof by showing that $x \in$ NE. Suppose not, then there is $j \in N$ and a best response $\tau_{j}^{\prime} \in \Sigma_{j}$ such that $u_{j}\left(\tau_{j}^{\prime}, x_{-j}\right)>u_{j}(x)$. As $G$ is a pseudopotential game, there is a best response $\tau_{j}^{*} \in \Sigma_{j}$ such that $P\left(\tau_{j}^{*}, x_{-j}\right)>P(x)$. Since $P$ is continuous, there is $\varepsilon>0$ such that for every $\tau \in B_{\varepsilon}\left(\left(\tau_{j}^{*}, x_{-j}\right)\right)$ it holds that $P(\tau)>$ $P(x)$. As $x^{\ell} \rightarrow x$, there is $\ell^{\prime} \in \mathbb{N}$ such that $x^{\ell^{\prime}} \in B_{\varepsilon}(x)$, so $\left(\tau_{j}^{*}, x_{-j}^{\ell^{\prime}}\right) \in B_{\varepsilon}\left(\left(\tau_{j}^{*}, x_{-j}\right)\right)$ and $P\left(\tau_{j}^{*}, x_{-j}^{\ell^{\prime}}\right)>P(x)$. We have that

$$
P(x)<P\left(\left(\tau_{j}^{*}, x_{-j}^{\ell^{\prime}}\right)\right) \leq P\left(b\left(s^{\ell^{\prime}}\right)\right) \leq P(x),
$$

a contradiction. Consequently, it holds that $x \in \mathrm{NE}$.

Q.E.D.

\section{REFERENCES}

Aliprantis, C. D., AND K. C. Border (2006): Infinite Dimensional Analysis—a Hitchhiker's Guide. Berlin: Springer-Verlag.[128]

ASHLAGI, I., Y. KANORIA, AND J. D. LeShNO (2017): "Unbalanced Random Matching Markets: The Stark Effect of Competition," Journal of Political Economy, 125, 69-98.[127]

Aumann, R. J. (1959): "Acceptable Points in General Cooperative $n$-Person Games," in Contributions to the Theory of Games, Vol. IV, ed. by A. W. Tucker and R. D. Luce. Princeton: Princeton University Press, 287324.[126]

BÉAL, S., E. RÉMILA, AND P. SOlAL (2013): "Accessibility and Stability of the Coalition Structure Core," Mathematical Methods of Operations Research, 78, 187-202.[132,134]

BALA, V., AND S. GOYAL (2000): "A Noncooperative Model of Network Formation,” Econometrica, 68, 11811229.[125]

BondAREVA, O. N. (1963): "Some Applications of Linear Programming Methods to the Theory of Cooperative Games," Problemy Kibernetiki, 10, 119-139.[112]

CHwE, M. S.-Y. (1994): "Farsighted Coalitional Stability," Journal of Economic Theory, 63, 299-325.[111,112]

DemuYnck, T., P. J.-J. Herings, R. D. SAulle, AND C. SeEl (2019): "Supplement to 'The Myopic Stable Set for Social Environments,", Econometrica Supplemental Material, 87, https://doi.org/10.3982/ ECTA14954. [113]

Dindoš, M., AND C. MezzeTti (2006): "Better-Reply Dynamics and Global Convergence to Nash Equilibrium in Aggregative Games," Games and Economic Behavior, 54, 261-292.[127] 
Dubey, P., O. HAimanko, AND A. ZAPeChelnyuK (2006): "Strategic Complements and Substitutes, and Potential Games," Games and Economic Behavior, 54, 77-94.[113,126,127]

DuTTA, B., AND S. Mutuswami (1997): "Stable Networks," Journal of Economic Theory, 76, 322-344.[125]

DutTA, B., AND R. VOHRA (2017): "Rational Expectations and Farsighted Stability," Theoretical Economics, 12, 1191-1227.[112]

DutTA, B., S. GHOSAL, AND D. RAY (2005): "Farsighted Network Formation,” Journal of Economic Theory, 122, 143-164.[112]

FRIEDMAN, J. W., AND C. MEZZETTI (2001): “Learning in Games by Random Sampling,” Journal of Economic Theory, 98, 55-84.[112,117,127]

Gale, D., AND L. S. Shapley (1962): “College Admissions and the Stability of Marriage," American Mathematical Monthly, 69, 9-15.[113,122-124,127]

Greenberg, J. (1990): The Theory of Social Situations: An Alternative Game-Theoretic Approach. Cambridge: Cambridge University Press. [112]

HARSANYI, J. C. (1974): "An Equilibrium-Point Interpretation of Stable Sets and a Proposed Alternative Definition," Management Science, 20, 1472-1495.[118]

HART, S., AND M. KURZ (1983): “Endogenous Formation of Coalitions,” Econometrica, 51, 1047-1064.[121, 122]

Herings, P. J. J., A. Mauleon, And V. VAnnetelbosch (2004): "Rationalizability for Social Environments," Games and Economic Behavior, 49, 135-156.[112]

(2009): "Farsightedly Stable Networks," Games and Economic Behavior, 67, 526-541.[112,125,126]

(2017): "Stable Sets in Matching Problems With Coalitional Sovereignty and Path Dominance," Journal of Mathematical Economics, 71, 14-19.[118,120]

(2018): "Stability of Networks Under Horizon-K Farsightedness," Economic Theory (forthcoming). DOI:10.1007/s00199-018-1119-7.[112]

JACKSON, M. O., AND A. VAN DEN NOUWELAND (2005): "Strongly Stable Networks," Games and Economic Behavior, 51, 420-444.[125]

JACKSON, M. O., AND A. WATTS (2002): “The Evolution of Social and Economic Networks,” Journal of Economic Theory, 106, 265-295.[112,113,126,127]

JACKSON, M. O., AND A. WOLINSKY (1996): "A Strategic Model of Social and Economic Networks," Journal of Economic Theory, 71, 44-74.[124,125]

JENSEN, M. K. (2010): "Aggregative Games and Best-Reply Potentials," Economic Theory, 43, 45-66.[127]

Kóczy, L. Á., AND L. LAUwERs (2004): “The Coalition Structure Core Is Accessible," Games and Economic Behavior, 48, 86-93.[113,120,121,127]

KNUTH, D. E. (1976): Marriages Stables. Montreal: Les Presses de l'Université de Montreal. [122,124,128]

KONISHI, H., AND D. RAY (2003): "Coalition Formation as a Dynamic Process," Journal of Economic Theory, 110, 1-41.[120,121]

LUCAS, W. F. (1968): “A Game With No Solution,” Bulletin of the American Mathematical Society, 74, 237239.[112]

(1992): "Von Neumann-Morgenstern Stable Sets," in Handbook of Game Theory, Vol. 1, ed. by R. J. Aumann and S. Hart. Amsterdam: Elsevier Science Publishers, 543-590.[112]

Milgrom, P., AND J. ROBERTS (1990): "Rationalizability, Learning and Equilibrium in Games With Strategic Complementarities," Econometrica, 58, 1255-1277.[113,127]

Monderer, D., AND L. S. Shapley (1996): "Potential Games," Games and Economic Behavior, 14, 124143.[127]

PAGe, F. H. JR., AND M. Wooders (2009): "Strategic Basins of Attraction, the Path Dominance Core, and Network Formation Games," Games and Economic Behavior, 66, 462-487.[112,118,125]

PAGE, F. H. JR., M. WoOders, AND S. KAMAT (2005): "Networks and Farsighted Stability," Journal of Economic Theory, 120, 257-269.[112]

RAY, D., AND R. Vohra (2014): "Coalition Formation,” in Handbook of Game Theory, Vol. 4, ed. by H. P. Young and S. Zamir. Amsterdam: Elsevier, 239-326.[120,121]

(2015): “The Farsighted Stable Set,” Econometrica, 83, 977-1011.[112,120]

Roth, A. E., AND E. PERANSOn (1999): "The Redesign of the Matching Market for American Physicians: Some Engineering Aspects of Economic Design," American Economic Review, 89, 748-780.[127]

Roth, A. E., AND J. H. VAnde VATE (1990): "Random Paths to Stability in Two-Sided Matching," Econometrica, 58, 1475-1480.[123]

SAWA, R. (2014): "Coalitional Stochastic Stability in Games, Networks and Markets," Games and Economic Behaviour, 88, 90-111.[112,119]

SCARF, H. (1967): “The Core of an N Person Game,” Econometrica, 35, 50-69.[112] 
SELTEN, R. (1970): Preispolitik der Mehrproduktenunternehmung in der statischen Theorie. Berlin: SpringerVerlag. [127]

SHAPLEY, L. S. (1967): “On Balanced Sets and Cores,” Naval Research Logistics Quarterly, 14, 453-460.[112]

SHAPLEY, L. S., AND H. SCARF (1974): “On Cores and Indivisibility,” Journal of Mathematical Economics, 1, 23-37.[122]

SHubIK, M. (1962): Game Theory in the Social Sciences: Concepts and Solutions. Cambridge, MA: MIT Press.[121]

TAMURA, A. (1993): “Transformation From Arbitrary Matchings to Stable Matchings," Journal of Combinatorial Theory, Series A, 62, 310-323.[124]

TOPKIS, D. (1979): "Equilibrium Points in Non-Zero Sum $n$-Person Submodular Games," SIAM Journal of Control and Optimization, 17, 773-787.[113,127]

van DeEmen, A. M. A. (1991): "A Note on Generalized Stable Sets," Social Choice and Welfare, 8, 255260.[118]

von Neumann, J., AND O. Morgenstern (1944): Theory of Games and Economic Behavior. Princeton, NJ: Princeton University Press.[118]

VoorneVeld, M. (2000): “Best-Response Potential Games," Economics Letters, 66, 289-295.[127]

XuE, L. (1998): “Coalitional Stability Under Perfect Foresight,” Economic Theory, 11, 603-627.[112]

\section{Co-editor Joel Sobel handled this manuscript.}

Manuscript received 22 December, 2016; final version accepted 4 September, 2018; available online 19 September, 2018. 